\title{
Simple analytic QCD model with perturbative QCD behavior at high momenta
}

\author{
Carlos Contreras $^{1}$, Gorazd Cvetič ${ }^{1,2}$, Olivier Espinosa*1, and Héctor E. Martínez ${ }^{1,3}$ \\ ${ }^{1}$ Department of Physics, Universidad Técnica Federico Santa María (UTFSM), Valparaíso, Chile \\ ${ }^{2}$ Valparaiso Center for Science and Technology, UTFSM, Chile \\ ${ }^{3}$ Physik Department, Technische Universität München, D-85748 Garching, Germany
}

(Dated: October 26, 2018)

\begin{abstract}
Analytic QCD models are those where the QCD running coupling has the physically correct analytic behavior, i.e., no Landau singularities in the Euclidean regime. We present a simple analytic QCD model in which the discontinuity function of the running coupling at high momentum scales is the same as in perturbative QCD (just like in the analytic QCD model of Shirkov and Solovtsov), but at low scales it is replaced by a delta function which parametrizes the unknown behavior there. We require that the running coupling agree to a high degree with the perturbative coupling at high energies, which reduces the number of free parameters of the model from four to one. The remaining parameter is fixed by requiring the reproduction of the correct value of the semihadronic tau decay ratio.

PACS numbers: 12.38.Aw,12.38.Cy,13.35.Dx,11.55.Hx
\end{abstract}

\section{INTRODUCTION}

The running coupling $a_{\mathrm{pt}}\left(Q^{2}\right) \equiv \alpha_{s}\left(Q^{2}\right) / \pi$ in perturbative QCD (pQCD) possesses Landau singularities at low momenta $Q^{2}\left(0<Q^{2}<\Lambda^{2}\right.$ where $\left.\Lambda^{2} \sim 0.1 \mathrm{GeV}^{2}\right)$, where $-q^{2} \equiv Q^{2}$ and $q$ being the typical momentum of the considered process. These singularities are then reflected in perturbative evaluation of spacelike physical observables $\mathcal{D}\left(Q^{2}\right)$ in terms of powers of $a\left(\kappa Q^{2}\right)$ (with $\kappa \sim 1$ ). On the other hand, in local field theories with causality 1 the observables $\mathcal{D}\left(Q^{2}\right)$ are analytic functions of $Q^{2}$ in the entire complex $Q^{2}$-plane, with the exception of the negative semiaxis regime $Q^{2} \leq-M_{\mathrm{thr}}^{2}$, where the threshold mass $M_{\mathrm{thr}} \sim 10^{-1} \mathrm{GeV}$ reflects the threshold for production of (light) mesons. If $\mathcal{D}\left(Q^{2}\right)$ is evaluated in terms of powers of $a\left(\mu^{2}\right)$ (with renormalization scale $\mu^{2}=\kappa Q^{2}, \kappa=$ const. $\sim$ 1 ), the aforementioned analytic behavior of the true $\mathcal{D}\left(Q^{2}\right)$ should be reflected in $a\left(\kappa Q^{2}\right)$. In perturbative QCD this is not the case, and the Landau singularities of perturbative coupling $a_{\mathrm{pt}}\left(Q^{2}\right)$ should thus be regarded as unphysical.

On the other hand, analytic QCD models have the running coupling $\mathcal{A}_{1}\left(Q^{2}\right)$ (instead of $a_{\mathrm{pt}}\left(Q^{2}\right)$ ) which reflects more correctly the analytic properties of $\mathcal{D}\left(Q^{2}\right)$. Nonperturbative studies of ghost-gluon vertex and ghost and gluon propagators, using Schwinger-Dyson equations [2] and lattice calculations [3], give QCD coupling $a\left(Q^{2}\right)$ with a finite value at $Q^{2}=0$ and without Landau singularities at positive $Q^{2}$. The minimal analytic (MA) model of Shirkov and Solovtsov [4 6] is the first and to date the most widely used of the analytic QCD models. It "minimally" modifies the perturbative coupling $a_{\mathrm{pt}}\left(Q^{2}\right)$, by eliminating the offending part of the discontinuity function of $a_{\mathrm{pt}}\left(Q^{2}\right)$ on the positive axis of $Q^{2}$-plane $\left(0<Q^{2} \leq \Lambda^{2}\right)$ and keeping its discontinuity on the nonpositive axis unchanged. This results in a new coupling $\mathcal{A}_{1}^{(\mathrm{MA})}\left(Q^{2}\right)$ analytic in the complex $Q^{2}$ plane with the exception of the nonpositive semiaxis $-\infty<Q^{2} \leq 0$. It turns out that, at high $Q^{2}$, the MA coupling differs from the perturbative one by terms $\mathcal{A}_{1}^{(\mathrm{MA})}\left(Q^{2}\right)-a_{\mathrm{pt}}\left(Q^{2}\right) \sim\left(\Lambda^{2} / Q^{2}\right)$. Afterwards, other analytic models for $\mathcal{A}_{1}\left(Q^{2}\right)$ have been presented in the literature 7-13, and they satisfy certain additional constraints at low and/or at high $Q^{2}$. For further literature and reviews of various analytic QCD models, see Refs. [14 16.

Most of the analytic QCD models suffer from one or both of the following problems:

1. Analytic QCD models with few free parameters usually cannot reproduce the experimental value of the semihadronic tau decay ratio $r_{\tau}$. For example, MA is a model with this problem, cf. Ref. 5, 17. The value of $r_{\tau}$ is at present the most precisely measured low-energy QCD observable, with the squared momentum of the process $s=\left|Q^{2}\right|=m_{\tau}^{2} \approx 3 \mathrm{GeV}^{2}$.

2. The deviation of the analytic coupling $\mathcal{A}_{1}\left(Q^{2}\right)$ from the perturbative coupling $a_{\mathrm{pt}}\left(Q^{2}\right)$, at high $Q^{2} \gg \Lambda^{2}$, can be appreciable: $\mathcal{A}_{1}\left(Q^{2}\right)-a_{\mathrm{pt}}\left(Q^{2}\right) \sim\left(\Lambda^{2} / Q^{2}\right)^{k}(k=1$, or $2, \ldots)$. This implies that such analytic QCD model gives nonperturbative contributions which are at least partly from the ultraviolet (UV) regime $\sim\left(\Lambda^{2} / Q^{2}\right)^{k}$. Such UV nonperturbative contributions contravene the Operator product expansion (OPE) philosophy of the ITEP (Institute of Theoretical and Experimental Physics) group [18, which stipulates that the nonperturbative contributions come only from the infrared (IR) regime.

* Deceased, September 14, 2010 
In Refs. 12, 13 two analytic QCD models are constructed such that the correct value of $r_{\tau}$ is reproduced; however, at high $Q^{2}$ the deviations from perturbative QCD are large, namely, $\mathcal{A}_{1}\left(Q^{2}\right)-a_{\mathrm{pt}}\left(Q^{2}\right) \sim\left(\Lambda^{2} / Q^{2}\right)^{k}$ with $k=1$. Further, in Refs. [12, 13] a method is presented for the construction of higher order analogs of powers $a_{\mathrm{pt}}^{n}\left(Q^{2}\right)$ for any analytic QCD model.

In Ref. 9] an analytic coupling $\mathcal{A}_{1}\left(Q^{2}\right)$ is constructed which comes close to fulfilling the abovementioned ITEPOPE condition, namely, it achieves $\mathcal{A}_{1}\left(Q^{2}\right)-a_{\mathrm{pt}}\left(Q^{2}\right) \sim\left(\Lambda^{2} / Q^{2}\right)^{k}$ with $k=3$ at high $Q^{2}$. However, the higher order analogs and the implications for the value of $r_{\tau}$ are not investigated in that reference.

In Refs. [19, 20], analytic coupling $\mathcal{A}_{1}\left(Q^{2}\right)$ is calculated from certain classes of beta-functions, and such $\mathcal{A}_{1}\left(Q^{2}\right)$ fulfills the OPE condition exactly, i.e., $\left|\mathcal{A}_{1}\left(Q^{2}\right)-a_{\mathrm{pt}}\left(Q^{2}\right)\right|<\left(\Lambda^{2} / Q^{2}\right)^{k}$ for all $k>0$ at high $Q^{2}$. However, in such models it turns out to be difficult to reproduce the correct value of $r_{\tau}$ [19, 20.

In this paper we present a relatively simple analytic QCD model which approximately fulfills the OPE condition (i.e., it merges with perturbative QCD to a high degree of accuracy at high $\left|Q^{2}\right|>10^{1} \mathrm{GeV}^{2}$ ) and, simultaneously, reproduces the correct value of $r_{\tau}$.

In Section II] we present the model, by motivating it first with a specific simple form of the discontinuity function $\rho_{1}(\sigma)=\operatorname{Im} \mathcal{A}_{1}\left(Q^{2}=-\sigma-i \epsilon\right)$ in a specific renormalization scheme $\left(\beta_{2}=\beta_{3}=\cdots=0\right)$. In Section III we fix the free parameter by requiring that the model reproduce the correct value of $r_{\tau}$. There we also present results of the model for the Bjorken polarized sum rule (BjPSR) $d_{\mathrm{Bj}}\left(Q^{2}\right)$ at low $Q^{2}$ and compare them with experimental values. In Section IV we present conclusions.

\section{MODEL DESCRIPTION}

The perturbative coupling $a_{\mathrm{pt}}\left(Q^{2}\right) \equiv \alpha_{s}\left(Q^{2}\right) / \pi$ has singularities (cut) along the real $Q^{2}$ axis at $Q^{2} \leq Q_{b}^{2}\left(\sim \Lambda^{2}\right)$ in the complex plane, where $Q_{b}^{2}$ is the branching point. Application of the Cauchy theorem gives the following dispersion relation for $a_{\mathrm{pt}}\left(Q^{2}\right)$ :

$$
a_{\mathrm{pt}}\left(Q^{2}\right)=\frac{1}{\pi} \int_{-Q_{b}^{2}-\eta}^{+\infty} d \sigma \frac{\rho_{1}^{(\mathrm{pt})}(\sigma)}{\left(\sigma+Q^{2}\right)}
$$

where $\rho_{1}^{(\mathrm{pt})}(\sigma)=\operatorname{Im} a_{\mathrm{pt}}\left(Q^{2}=-\sigma-i \epsilon\right)$ is the perturbative discontinuity function, and $\eta \rightarrow+0$. The minimal analytic (MA) model of Shirkov and Solovtsov [4 6] consists of removing the offending cut at positive $Q^{2}\left(0<Q^{2} \leq Q_{b}^{2}\right)$, i.e., for $-Q_{b}^{2} \leq \sigma<0$

$$
\mathcal{A}_{1}^{(\mathrm{MA})}\left(Q^{2}\right)=\frac{1}{\pi} \int_{0}^{+\infty} d \sigma \frac{\rho_{1}^{(\mathrm{pt})}(\sigma)}{\left(\sigma+Q^{2}\right)}
$$

In analogous way, the higher order couplings $\mathcal{A}_{n}(n=2,3, \ldots)$ are constructed in MA analogously ([5, 6, 15] and references therein $)^{1}$

$$
\mathcal{A}_{n}^{(\mathrm{MA})}\left(Q^{2}\right)=\frac{1}{\pi} \int_{0}^{+\infty} d \sigma \frac{\rho_{n}^{(\mathrm{pt})}(\sigma)}{\left(\sigma+Q^{2}\right)}
$$

where $\rho_{n}^{(\mathrm{pt})}(\sigma)=\operatorname{Im} a_{\mathrm{pt}}^{n}\left(Q^{2}=-\sigma-i \epsilon\right)$.

As mentioned before, once the parameter $\bar{\Lambda}$ (the $\overline{\mathrm{MS}} \Lambda$-scale) is adjusted in MA so that the high-energy QCD phenomenology is reproduced $\left(\bar{\Lambda} \approx 400-440 \mathrm{MeV}\right.$ when $n_{f}=3$, Ref. [6] $)$, the value of the semihadronic tau decay ratio $r_{\tau}$ (strangeless and massless) is predicted to be about 0.14, much lower [13, 17, than the well measured experimental value $r_{\tau} \approx 0.203 \pm 0.004$. Another possibly unattractive aspect of MA is that its coupling $\mathcal{A}_{1}^{(\mathrm{MA})}\left(Q^{2}\right)$ has singularities along the entire nonpositive $Q^{2}$ axis $\left(Q^{2} \leq 0\right.$, i.e., $\left.\sigma \geq 0\right)$ in the complex $Q^{2}$-plane. This does not reflect closely the analyticity properties of spacelike observables $\mathcal{D}\left(Q^{2}\right)$ which have nonanalyticity cut along negative $Q^{2}$ axis starting at a negative threshold value $-M_{\mathrm{thr}}^{2}: Q^{2} \leq-M_{\mathrm{thr}}^{2}$ (i.e., $\sigma \geq M_{\mathrm{thr}}^{2}$ ). The value of the threshold mass $M_{\mathrm{thr}}$ is typically a (multiple of) mass of light mesons. One possibility to incorporate such behavior in the analytic coupling is to

1 The MA couplings $\mathcal{A}_{n}^{(\mathrm{MA})}(n \geq 1)$ defined here are the MA couplings of Refs. 4, 6] 15] divided by $\pi$. 
eliminate certain IR interval $0 \leq \sigma<M_{\mathrm{thr}}^{2}$ of the cut in the dispersive relation 2), resulting in a "modified" MA (mMA) coupling

$$
\mathcal{A}_{1}^{(\mathrm{mMA})}\left(Q^{2}\right)=\frac{1}{\pi} \int_{M_{\mathrm{thr}}^{2}}^{+\infty} d \sigma \frac{\rho_{1}^{(\mathrm{pt})}(\sigma)}{\left(\sigma+Q^{2}\right)} .
$$

Such type of change was proposed in Refs. 8..$^{2}$ In Ref. 21, it was pointed out that the coupling (4) is a Stieltjes function, and that, as a consequence, the paradiagonal Padé approximants $[M-1 / M]\left(Q^{2}\right)$ of such a coupling systematically converge to the exact values $\mathcal{A}_{1}^{(\mathrm{mMA})}\left(Q^{2}\right)$ when the Padé index $M$ increases. ${ }^{3}$ The latter fact was checked numerically, and it was also shown there that the aforementioned Padé approximations are equivalent to approximating the mMA discontinuity function by a sum of $M$ delta terms

$$
\begin{aligned}
\frac{1}{\pi} \rho_{1}^{(\mathrm{mMA})}(\sigma) & =\Theta\left(\sigma-M_{\mathrm{thr}}^{2}\right) \times \operatorname{Im} a_{\mathrm{pt}}\left(Q^{2}=-\sigma-i \epsilon\right) \\
& \approx \sum_{n=1}^{M} f_{n}^{2} \Lambda^{2} \delta\left(\sigma-M_{n}^{2}\right)=\sum_{n=1}^{m} f_{n}^{2} \delta\left(s-s_{n}\right),
\end{aligned}
$$

where $s=\sigma / \Lambda^{2}, s_{n}=M_{n}^{2} / \Lambda^{2}$ and $f_{n}$ are positive dimensionless quantities, and $M_{\mathrm{thr}} \approx M_{1}<M_{2}<\cdots$. Therefore, it was argued that, although a sum of delta functions appears to be a very crude approximation for the (continuous) function $\rho_{1}(\sigma)$, it gives increasingly better expressions for the coupling $\mathcal{A}_{1}\left(Q^{2}\right)$ when the number $M$ of deltas in the sum 6 increases. We only know the approximate behavior of the true $\rho_{1}(\sigma)$ at high $\sigma \gg \Lambda^{2}\left[\rho_{1}(\sigma) \approx \rho_{1}^{(\mathrm{pt})}(\sigma)\right]$, and we do not know the behavior in the IR regime $\sigma \sim \Lambda^{2}$. Therefore, as was also argued in Ref. [21], in the regime of low positive $\sigma$ we can expect that parametrization of the true $\rho_{1}(\sigma)$ in terms of one or a few delta functions may lead to a reasonably realistic behavior of $\mathcal{A}_{1}\left(Q^{2}\right)$ at low $\left|Q^{2}\right|$. In this work we use only one delta function in the IR regime of $\sigma^{\prime}$ s:

$$
\begin{aligned}
\rho_{1}(\sigma) & =\pi f_{1}^{2} \Lambda^{2} \delta\left(\sigma-M_{1}^{2}\right)+\Theta\left(\sigma-M_{0}^{2}\right) \times \rho_{1}^{(\mathrm{pt})}(\sigma) \\
& =\pi f_{1}^{2} \delta\left(s-s_{1}\right)+\Theta\left(s-s_{0}\right) \times r_{1}^{(\mathrm{pt})}(s)
\end{aligned}
$$

where $s=\sigma / \Lambda^{2}, s_{1}=M_{1}^{2} / \Lambda^{2}, s_{0}=M_{0}^{2} / \Lambda^{2}$, and $r_{1}^{(\mathrm{pt})}(s)=\rho_{1}^{(\mathrm{pt})}(\sigma)=\operatorname{Im} a_{\mathrm{pt}}\left(Q^{2}=-\sigma-i \epsilon\right)$. We may expect ${ }^{4}$ $0<M_{1}<M_{0}$, i.e., the actual threshold mass is $M_{1}$. The discontinuity function $\rho_{1}(\sigma)$, Eq. (7), is depicted in Figs. 1 . (a), (b), for the choice of two sets of values of the parameters $\left(M_{1}, M_{0}\right.$ and $\Lambda$ ), which will be motivated in the next Section.
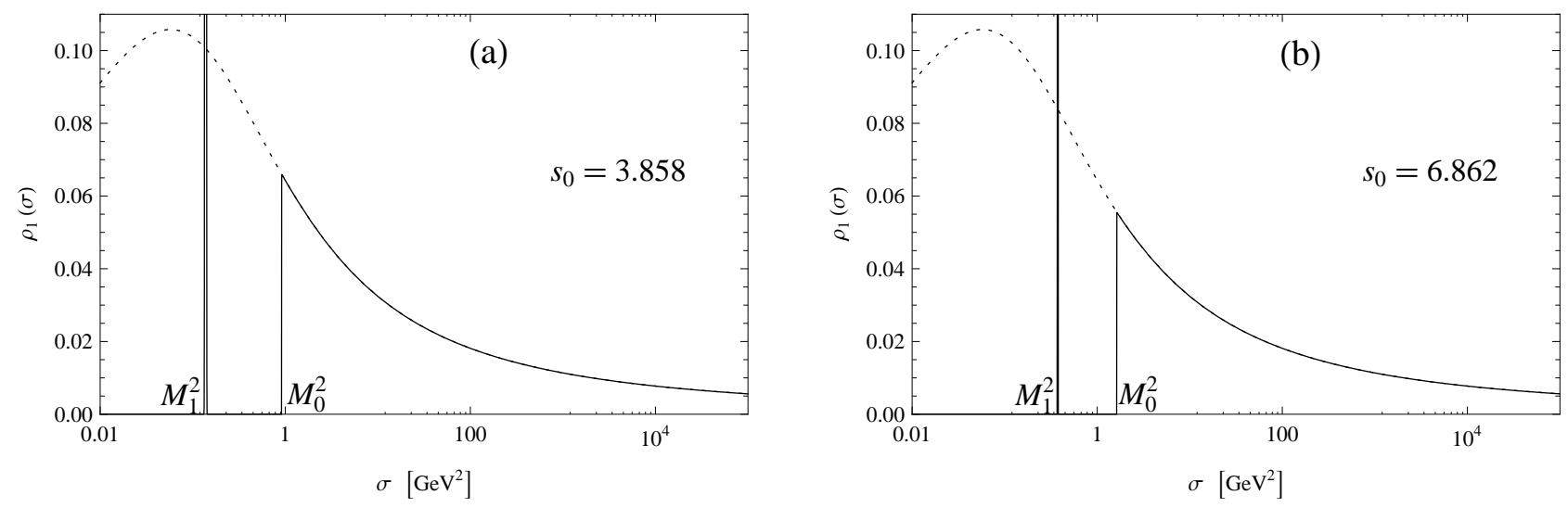

2 The authors of [8] used for $\mathcal{A}_{1}$, before eliminating an IR-cut, a "second" MA model [7, which is obtained by minimal analytization of the right-hand side of the following form of the perturbative renormalization group equation (RGE): $d \ln a / d \ln Q^{2}=-\beta_{0} a-\beta_{1} a^{2}-\beta_{2} a^{2}-\cdots$. Their numerical analysis is then performed at the one-loop level.

3 Padé approximant $[N / M](x)$ to a function $f(x)$ is, by definition, a ratio $P_{N}(x) / Q_{M}(x)$ of polynomials of degree $N$ and $M$, respectively, such that the Taylor expansions of $[N / M](x)$ and of $f(x)$ around $x=0$ agree up to (and including) the term $\sim x^{N+M}$.

4 Although we do not impose the condition $0<M_{1}<M_{0}$, it will turn out to be true in our model (see the next Section). 
FIG. 1: The discontinuity function $\rho_{1}(\sigma)$, Eq. (7), for the values of scale parameters $\Lambda=0.487 \mathrm{GeV}$ and: (a) $M_{1}=0.371 \mathrm{GeV}$ and $M_{0}=0.956 \mathrm{GeV}\left(s_{1} \equiv M_{1}^{2} / \Lambda^{2}=0.581\right.$ and $\left.s_{0} \equiv M_{0}^{2} / \Lambda^{2}=3.858\right) ;(\mathrm{b}) M_{1}=0.612 \mathrm{GeV}$ and $M_{0}=1.275 \mathrm{GeV}\left(s_{1} \equiv M_{1}^{2} / \Lambda^{2}=1.579\right.$ and $\left.s_{0} \equiv M_{0}^{2} / \Lambda^{2}=6.862\right)$. For comparison, perturbative discontinuity function $\rho_{1}^{\mathrm{pt}}(\sigma)$ at positive $\sigma$ is also included as a dotted curve [in $\beta_{2}=\beta_{3}=\cdots=0$ renormalization scheme (RSch), and with $\left.n_{f}=3\right]$.

A physical interpretation of the ansatz (7)-(8) is also possible: If the discontinuity function $\rho_{1}(\sigma)$ is to simulate, in the first approximation, the spectral functions $\rho_{\mathcal{D}}(\sigma)=\operatorname{Im} \mathcal{D}\left(Q^{2}=-\sigma-i \epsilon\right)$ of spacelike observables $\mathcal{D}\left(Q^{2}\right)$, the delta-term in Eqs. (7)-(8) and in Figs. 1 can be regarded as a narrow width approximation (NWA) of the dominant lowenergy resonance. ${ }^{5}$ For an application of NWA ansatz directly to the spectral function of the vector current-current correlator ( $\Leftrightarrow$ Adler function), see Ref. 22 .

Furthermore, in a recent work of Ref. 23] a similar idea was applied directly to the spectral function $v_{1}(\sigma)$ of the vector current-current correlator. The ansatz for $v_{1}(\sigma)$ there is similar to the ansatz (7)-(8). However, instead of approximating the low-energy regime $\left(\sigma<M_{0}^{2}\right)$ by a simple delta function, the known measured low-energy values of $v_{1}(\sigma)$ of the ALEPH and OPAL collaborations [24 26] were used. The threshold value of the onset of perturbative QCD $\left(M_{0}^{2}\right)$, and the perturbative coupling strength $(\Leftrightarrow \bar{\Lambda})$ were then fixed by two conditions: the correct measured value of the (strangeless) $r_{\tau}$ has to be reproduced, and the higher-twist terms of the resulting Adler function have no terms of dimension two $\left(\propto 1 / Q^{2}\right)$. The work of Ref. [23] is a generalization and refinement of an earlier work Ref. 27.

The dispersion relation applied to the discontinuity function (7)-(8) then gives us the following expression for the analytic coupling $\mathcal{A}_{1}\left(Q^{2}\right)$ :

$$
\mathcal{A}_{1}\left(Q^{2}\right)=\frac{1}{\pi} \int_{0}^{+\infty} d \sigma \frac{\rho_{1}(\sigma)}{\left(\sigma+Q^{2}\right)}=\frac{f_{1}^{2}}{u+s_{1}}+\frac{1}{\pi} \int_{s_{0}}^{\infty} d s \frac{r_{1}^{(\mathrm{pt})}(s)}{(s+u)}
$$

where we use the notation

$$
u=Q^{2} / \Lambda^{2}, \quad s=\sigma / \Lambda^{2} .
$$

In the renormalization scheme (RSch) with the beta function coefficients $\beta_{2}=\beta_{3}=\cdots=0$, the discontinuity function is explicitly known in terms of Lambert function $W_{ \pm 1}$, Ref. [28] (see also Refs. [29, 30] on the use of Lambert functions in evaluation of the $n$-loop coupling)

$$
\begin{aligned}
r_{1}^{(\mathrm{pt})}(s) & =\operatorname{Im}\left[\frac{1}{c_{1}} \frac{1}{\left[1+W_{-1}\left(\frac{1}{c_{1} e} s^{-\beta_{0} / c_{1}} \exp \left[-i \pi\left(\beta_{0} / c_{1}-1\right)\right]\right)\right]}\right] \\
& =\operatorname{Im}\left[\frac{-1}{c_{1}} \frac{1}{\left[1+W_{+1}\left(\frac{1}{c_{1} e} s^{-\beta_{0} / c_{1}} \exp \left[+i \pi\left(\beta_{0} / c_{1}-1\right)\right]\right)\right]}\right],
\end{aligned}
$$

where $s=\sigma / \Lambda^{2}$, and $\beta_{0}$ and $c_{1}=\beta_{1} / \beta_{0}$ are the two (universal) coefficients in the renormalization group equation in the aforementioned renormalization scheme

$$
\frac{d a\left(Q^{2}\right)}{d \ln Q^{2}}=-\beta_{0} a^{2}\left(1+c_{1} a\right)
$$

specifically $\beta_{0}=\left(11-2 n_{f} / 3\right) / 4$ and $\beta_{1}=\beta_{0} c_{1}=\left(102-38 n_{f} / 3\right) / 16$. It is in this renormalization scheme that we will asume the form $\sqrt{8}$ ) of the discontinuity function, and consequently, the form $(9)$ of $\mathcal{A}_{1}\left(Q^{2}\right)$. The $\Lambda$ scale appearing implicitly in these expressions is the Lambert $\Lambda$, and it is related with the $\overline{\mathrm{MS}}$ scale $\bar{\Lambda}$ at $n_{f}=3$ via

$$
\Lambda \approx \bar{\Lambda} \exp (0.3205)
$$

The coupling $a_{\mathrm{pt}}\left(Q^{2}\right)$ in the $\beta_{2}=\beta_{3}=\cdots=0$ renormalization scheme has a Landau cut along $0<Q^{2}<Q_{b}^{2}$, where $Q_{b}^{2}$ is the branching point $Q_{b}^{2}=\Lambda^{2} s_{b}$, where $s_{b}=c_{1}^{-c_{1} / \beta_{0}} \approx 0.635$ when $n_{f}=3$. The perturbative coupling $a_{\mathrm{pt}}\left(Q^{2}\right)$ in this renormalization scheme is known 28

$$
a_{\mathrm{pt}}\left(Q^{2}\right)=-\frac{1}{c_{1}} \frac{1}{\left[1+W_{\mp 1}\left(z_{ \pm}\right)\right]},
$$

\footnotetext{
5 For two simpler-minded analytic QCD models involving delta-function in $\rho_{1}(\sigma)$, see Refs. [12, 13].
} 
where $Q^{2}=\left|Q^{2}\right| \exp (i \phi)$, the upper signs refer to the case $0 \leq \phi<+\pi$, the lower signs to $-\pi<\phi<0$, and

$$
z_{ \pm}=\frac{1}{c_{1} e}\left(\frac{\left|Q^{2}\right|}{\Lambda^{2}}\right)^{-\beta_{0} / c_{1}} \exp \left[i\left( \pm \pi-\frac{\beta_{0}}{c_{1}} \phi\right)\right]
$$

It can be numerically checked that the dispersion relation $(1)$ holds for $a_{\mathrm{pt}}\left(Q^{2}\right)$ in this renormalization scheme, using for the discontinuity function the expressions (11) at $s \equiv \sigma / \Lambda^{2}>0$, and for $-s_{b}<s \equiv \sigma / \Lambda^{2}<0$ the expression

$$
r_{1}^{(\mathrm{pt})}(s)=\operatorname{Im}\left[\frac{1}{c_{1}} \frac{1}{\left[1+W_{-1}\left(\frac{-1}{c_{1} e}|s|^{-\beta_{0} / c_{1}}+i \epsilon\right)\right]}\right] .
$$

One peculiar feature in most of the analytic QCD models is that at large $\left|Q^{2}\right|\left(\gg \Lambda^{2}\right)$ the analytic coupling $\mathcal{A}_{1}\left(Q^{2}\right)$ differs from the perturbative coupling $a_{\mathrm{pt}}\left(Q^{2}\right)$ by an inverse power of $Q^{2}$ :

$$
\left|\mathcal{A}_{1}\left(Q^{2}\right)-a_{\mathrm{pt}}\left(Q^{2}\right)\right| \sim\left(\frac{\Lambda^{2}}{Q^{2}}\right)^{k} \quad\left(\left|Q^{2}\right| \gg \Lambda^{2}\right) .
$$

In MA [4, 5], and in the "second MA" of Refs. [7, this power is $k=1$, i.e., relatively large deviation.

However, power deviations (17) are not in accordance with the philosophy of the Operator product expansion (OPE) as promoted by the ITEP group [18. According to that philosophy, all nonperturbative contributions such as $\left(\Lambda^{2} / Q^{2}\right)^{k}$ to (inclusive) observables originate from the infrared (IR) regimes $\left|Q^{2}\right| \lesssim \Lambda^{2}$. Consequently, the OPE of spacelike inclusive observables is interpreted in this approach as superposition of perturbative contributions coming from the ultraviolet regime $\left|Q^{2}\right| \gg \Lambda^{2}$ (Wilson coefficients) and nonperturbative contributions coming from the IR regime (vacuum expectation values of operators). Once we have power deviations of the type (17), this implies that a theory with such $\mathcal{A}_{1}\left(Q^{2}\right)$ will give us, in evaluations of inclusive spacelike observables, nonperturbative terms (of the type $\Lambda^{2} / Q^{2}$ ) coming at least partly from the UV regime, thus contravening the ITEP philosophy.

More specifically, the authors of Ref. 31] argued in the following way that the terms $\sim\left(\Lambda^{2} / Q^{2}\right)^{k}$ in the deviation Eq. 17) contravene the ITEP philosophy of OPE. Namely, let us consider the leading- $\beta_{0}$ summation of an inclusive spacelike observable $\mathcal{D}\left(Q^{2}\right)$

$$
\mathcal{D}^{(\mathrm{LB})}\left(Q^{2}\right) \equiv \int_{0}^{\infty} \frac{d t}{t} F_{\mathcal{D}}(t) a\left(t Q^{2} e^{\overline{\mathcal{C}}}\right)
$$

Here, $F_{\mathcal{D}}(t)$ is the characteristic function of the observable and $\overline{\mathcal{C}}=-5 / 3$. The quantity $t Q^{2} e^{\overline{\mathcal{C}}}$ is the square of internal loop momenta appearing in the resummation. In the UV regime $t>1$, the deviation 17) then produces power term contributions of UV origin in the observable (see also Ref. [32])

$$
\delta \mathcal{D}^{(\mathrm{LB})}\left(Q^{2}\right) \sim\left(\Lambda^{2} / Q^{2}\right)^{k} \int_{1}^{\infty} \frac{d t}{t^{k+1}} F_{\mathcal{D}}(t) \sim\left(\Lambda^{2} / Q^{2}\right)^{k} .
$$

For more discussion on these aspects, we refer to Ref. 20. The aforementioned OPE condition then implies

$$
\left|\mathcal{A}_{1}\left(Q^{2}\right)-a_{\mathrm{pt}}\left(Q^{2}\right)\right|<\left(\frac{\Lambda^{2}}{Q^{2}}\right)^{k} \quad\left(\left|Q^{2}\right| \gg \Lambda^{2} ; k=1,2, \ldots\right)
$$

This condition can be interpreted as a requirement that the analytic QCD model be formally perturbative (analytic) QCD at high momenta. We want to construct here a simple analytic QCD model with the discontinuity function $\rho_{1}(\sigma)$ of the type (7) which is as close as possible to perturbative QCD (in renormalization scheme $\beta_{2}=\beta_{3}=\cdots=0$ ). In such a model, all the measured values of the high-energy QCD observables (with $\left|Q^{2}\right|>10^{1} \mathrm{GeV}^{2}$ ) are then reproduced just as in perturbative QCD. In such a model, we have to ensure only that the values of the well measured low-energy observables, particularly $r_{\tau}$ (with $\left|Q^{2}\right|=m_{\tau}^{2} \approx 3 \mathrm{GeV}^{2}$ ), are reproduced.

It turns out to be difficult to construct analytic QCD theories which fully respect the condition $[20$, , see Refs. $19,20.2$ We will construct here an analytic QCD model of Eqs. (7)-(9), which only approximately fulfills the condition (20). We will end up with a simple analytic QCD model where the number of free parameters is reduced to one, and the latter parameter will be fixed by imposing the condition of the reproduction of the correct value of $r_{\tau}$. We note that the model of Eqs. (7)-(9) at first contains three free dimensionless positive parameters $\left(f_{1}^{2}, s_{1}, s_{0}\right)$, in addition to the energy scale parameter $\Lambda$. The parameters $f_{1}^{2}, s_{1}$ and the scale $\Lambda$ will be fixed by requiring: the condition that $k=3$ 
in the relation Eq. (17) instead ${ }^{6}$ of $k=1$; and the correct perturbative QCD value of $\mathcal{A}_{1}\left(Q^{2}\right)$ at $Q^{2}=\left(3 m_{c}\right)^{2}$. This is approximately the highest value of $Q^{2}$ where the number of active quark flavors can still reasonably safely be kept $n_{f}=3$ in perturbative QCD with four-loop RGE-running and three-loop matching conditions $33 .{ }^{7}$

A consistent accounting of the threshold effects fully within analytic QCD, when $n_{f} \mapsto\left(n_{f}+1\right)$, is not yet known for general analytic QCD models. However, in MA of Refs. 4, 5], such an accounting can be made systematically in the following way (see Refs. [6], and also Refs. [35): At the positive threshold value $Q_{\mathrm{thr}}^{2}=\left(\kappa m_{c}\right)^{2}$, with $\kappa \sim 1$ (usually in perturbative QCD: $1 \leq \kappa \leq 3$ ), the perturbative coupling can be taken to be continuous to a high degree of accuracy: $a_{\mathrm{pt}}\left(Q_{\mathrm{thr}}^{2} ; n_{f}=3\right)=\bar{a}_{\mathrm{pt}}\left(\bar{Q}_{\mathrm{thr}}^{2} ; n_{f}=4\right)$. The latter condition then relates the scale parameters $\bar{\Lambda}\left(n_{f}=3\right)$ and $\bar{\Lambda}\left(n_{f}=4\right)$. In MA, the discontinuity function is then constructed as $\rho_{1}^{(\mathrm{MA})}(\sigma)=\operatorname{Im} a_{\mathrm{pt}}\left(Q^{2}=-\sigma-i \epsilon ; n_{f}\right)$ with $n_{f}=3$ for $\sigma<\left(\kappa m_{c}\right)^{2}$, and $n_{f}=4$ for $\left(\kappa m_{c}\right)^{2}<\sigma<\left(\kappa m_{b}\right)^{2}$. Analogous threshold effects are implemented in $\rho_{1}^{(\mathrm{MA})}$ for the transition $n_{f}=4 \mapsto 5$ at the threshold $\sigma_{\mathrm{thr}}=\left(\kappa m_{b}\right)^{2}$. The function $\rho_{1}^{(\mathrm{MA})}(\sigma)$ is thus step-like discontinuous. The resulting (global) MA coupling $\mathcal{A}_{1}\left(Q^{2}\right)$, constructed via the dispersion relation 2 with $\rho_{1}^{(\mathrm{pt})}$ replaced by the aforementioned step-wise discontinuous $\rho_{1}^{\text {(MA) }}$, remains analytic in the entire Euclidean region $Q^{2} \in \mathbb{C} \backslash(-\infty, 0]$. The values of $\bar{\Lambda}\left(n_{f}\right)$ 's can then be fixed by requiring that the model reproduce the measured values of high-energy QCD observables (e.g., with $\left|Q^{2}\right|>10^{1} \mathrm{GeV}^{2}$ ).

This threshold matching procedure could be implemented also in the present model (in the renormalization scheme $\left.\beta_{2}=\beta_{3}=\cdots=0\right)$ by making $\rho_{1}(\sigma)$ accordingly step-like discontinuous at $\sigma_{\text {thr }}$ 's. However, by assuming that at $Q_{\mathrm{thr}}^{2}=\left(3 m_{c}\right)^{2}$ the present $n_{f}=3$ analytic QCD model merges with perturbative QCD does not result in any appreciable error, due to the condition that in the relation (17) we have $k=3$ [see also the comments later in the text, the paragraph just after Eq. (25) and the paragraph at Eq. (39p].

Throughout this work we assume that we are in the regime of three active quark flavors $\left(n_{f}=3\right)$, and that the three quarks $u, d$ and $s$ are (almost) massless. The "approximate perturbative QCD" condition $k=3$ in Eq. (17) can be expressed via the following two conditions:

$$
\begin{gathered}
\frac{1}{\pi} \int_{-s_{b}}^{+s_{0}} d s r_{1}^{(\mathrm{pt})}(s)=f_{1}^{2}, \\
\frac{1}{\pi} \int_{-s_{b}}^{+s_{0}} d s s r_{1}^{(\mathrm{pt})}(s)=s_{1} f_{1}^{2},
\end{gathered}
$$

where $s_{b}=c_{1}^{-c_{1} / \beta_{0}}=Q_{b}^{2} / \Lambda^{2}, Q_{b}^{2}$ being the branch point of $a_{\mathrm{pt}}\left(Q^{2}\right)$ in the complex $Q^{2}$-plane. Conditions 21 - 22 mean that the coefficient of $\left(Q^{2} / \Lambda^{2}\right)$ and $\left(Q^{2} / \Lambda^{2}\right)^{2}$ in the deviation $\mathcal{A}_{1}\left(Q^{2}\right)-a_{\mathrm{pt}}\left(Q^{2}\right)$ is zero, respectively. Equations (21)-22) were obtained by subtracting Eq. (9) from Eq. (1)

$$
a_{\mathrm{pt}}\left(Q^{2}\right)-\mathcal{A}_{1}\left(Q^{2}\right)=-\frac{f_{1}^{2}}{u+s_{1}}+\frac{1}{\pi} \int_{-s_{b}}^{+s_{0}} d s \frac{r_{1}^{(\mathrm{pt})}(s)}{(s+u)},
$$

and expanding in powers of $(1 / u)=\left(\Lambda^{2} / Q^{2}\right)$.

In addition to the two conditions (21)- 22 , there is a condition that the theory merge with the perturbative coupling (in the renormalization scheme $\beta_{2}=\beta_{3}=\cdots=0$ ) at higher renormalization scales $\mu^{2}$. Since we do not yet know a consistent exact threshold conditions within analytic QCD models, we assume that our analytic QCD model coupling $\mathcal{A}_{1}\left(\mu^{2}\right)$ has the number of active quarks $n_{f}=3$ up to the renormalization scale $(\mathrm{RScl}) \mu^{2}=\left(3 m_{c}\right)^{2}$ (with $m_{c} \approx 1.27 \mathrm{GeV}$ ) and that at that scale it merges with the value of the perturbative coupling $a_{\mathrm{pt}}\left(\left(3 m_{c}\right)^{2} ; \beta_{2}=\right.$ $\left.\beta_{3}=\cdots=0 ; n_{f}=3\right)$ such as implied by the high energy QCD experiments. Specifically, high energy QCD implies $a\left(M_{Z}^{2}, \overline{\mathrm{MS}}\right) \approx 0.119 / \pi$, Ref. [34. We then run this value, by perturbative four-loop RGE in $\overline{\mathrm{MS}}$ renormalization scheme, down to renormalization scale $\mu^{2}=\left(3 m_{c}\right)^{2}$, and incorporate quark thresholds at $\mu^{2}=\left(3 m_{q}\right)^{2}(q=b, c)$ by three-loop matching conditions Ref. [33. We obtain in this way $\bar{a}_{\mathrm{pt}} \equiv a_{\mathrm{pt}}\left(\left(3 m_{c}\right)^{2}, \overline{\mathrm{MS}}, n_{f}=3\right)=0.07245$. Changing renormalization scheme from $\overline{\mathrm{MS}}$ to $\beta_{2}=\cdots=0$ by the subtracted form (Ref. [36], Appendix A there) of the

6 This condition, with $k=3$, is also fulfilled in the model for $\mathcal{A}_{1}\left(Q^{2}\right)$ of Ref. $[9]$.

7 If we apply the four-loop RGE-running in $\overline{\mathrm{M} S}$ renormalization scheme with three-loop matching conditions according to Ref. 33 at $Q^{2}=\left(\kappa m_{c}\right)^{2}$ and $Q^{2}=\left(\kappa m_{b}\right)^{2}$, and choose a fixed initial value $a\left(m_{c}^{2}, \overline{\mathrm{MS}} ; n_{f}=3\right)=0.12945$ at the initial $Q_{0}^{2}=m_{c}^{2}[$ which gives: $a\left(\left(3 m_{c}\right)^{2}, \overline{\mathrm{MS}} ; n_{f}=3\right)=0.07245$, i.e., the value we use in this paper], we obtain the values $\alpha_{s}\left(M_{Z}^{2}, \overline{\mathrm{MS}}\right)=0.1190,0.1191,0.1193$, when choosing for the threshold parameter $\kappa$ the values $\kappa=3,2,1$, respectively. For the quark masses we use the values $m_{c}=1.27 \mathrm{GeV}$ and $m_{b}=4.20 \mathrm{GeV}$ 34. 
TABLE I: The dimensionless nonnegative parameters $s_{1}=M_{1}^{2} / \Lambda^{2}$ and $f_{1}^{2}$ as functions of the cutoff parameter $s_{0}=M_{0}^{2} / \Lambda^{2}$ (>0). The scale $\Lambda$ is practically independent of $s_{0}: \Lambda \approx 0.487 \mathrm{GeV}$. The penultimate column shows the leading- $\beta_{0}$ resummed (LB) contributions to $r_{\tau}$; in parentheses the leading order (LO) contribution. The last column shows the sum of the LB and the beyond-the-leading- $\beta_{0}$ contribution ( $\left.\mathrm{LB}+\mathrm{bLB}\right)$ to $r_{\tau}$; in parentheses the $\mathrm{LO}$ and beyond-leading-order contribution $(\mathrm{LO}+\mathrm{bLO})$ to $r_{\tau}$ (for details on $r_{\tau}$, see the next section).

\begin{tabular}{lllll}
\hline \hline$s_{0}$ & $s_{1}$ & $f_{1}^{2}$ & $r_{\tau}^{(\mathrm{LB})}\left(r_{\tau}^{(\mathrm{LO})}\right)$ & $r_{\tau}^{(\mathrm{LB}+\mathrm{bLB})}\left(r_{\tau}^{(\mathrm{LO}+\mathrm{bLO})}\right)$ \\
\hline 1.958 & 0.0000 & 0.1721 & $0.2522(0.1315)$ & $0.2399(0.1892)$ \\
2.000 & 0.0121 & 0.1732 & $0.2509(0.1315)$ & $0.2386(0.1893)$ \\
3.000 & 0.3117 & 0.1970 & $0.2290(0.1316)$ & $0.2166(0.1915)$ \\
3.858 & 0.5812 & 0.2156 & $0.2156(0.1317)$ & $0.2030(0.1939)$ \\
4.000 & 0.6267 & 0.2186 & $0.2137(0.1317)$ & $0.2010(0.1943)$ \\
5.000 & 0.9523 & 0.2387 & $0.2016(0.1319)$ & $0.1885(0.1975)$ \\
6.000 & 1.2861 & 0.2576 & $0.1916(0.1321)$ & $0.1781(0.2006)$ \\
6.862 & 1.5788 & 0.2732 & $0.1844(0.1323)$ & $0.1704(0.2030)$ \\
\hline \hline
\end{tabular}

integrated perturbative QCD RGE (see Ref. [37, Appendix A there) then results in ${ }^{8}$

$$
a_{\mathrm{in}} \equiv a_{\mathrm{pt}}\left(\left(3 m_{c}\right)^{2} ; \beta_{2}=0, \beta_{3}=0, \ldots ; n_{f}=3\right)=0.07050 .
$$

As stated above, we require that $\mathcal{A}_{1}\left(\mu^{2}\right)$ of our analytic QCD model, at $\mu^{2}=\left(3 m_{c}\right)^{2}$, agrees with the value Eq. 24, i.e., the analytic QCD model merges with perturbative QCD starting at the scale $\mu^{2}=\left(3 m_{c}\right)^{2}$ upwards

$$
\mathcal{A}_{1}\left(\mu^{2}=\left(3 m_{c}\right)^{2}\right)=a_{\text {in }} \quad(=0.07050) .
$$

One may worry that the replacement of the presented analytic QCD model by perturbative QCD at $Q^{2} \geq\left(3 m_{c}\right)^{2}$ [and with $n_{f}=3 \mapsto 4$ perturbative threshold at $Q^{2}=\left(3 m_{c}\right)^{2}$ ] may not be a good approximation, i.e., that the analytic coupling $\mathcal{A}_{1}\left(Q^{2}\right)$ of the theory, say at fixed $n_{f}=3$, behaves at high scales $Q^{2} \sim M_{Z}^{2}$ significantly different than the perturbative coupling $a_{\mathrm{pt}}\left(Q^{2}\right)$. It turns out that this is not the case. Namely, if we formally keep fixed $n_{f}=3$ (in order not to worry about threshold effects in analytic QCD), the perturbative RGE-running (in the renormalization scheme $\left.\beta_{2}=\cdots=0\right)$ from the initial value $a_{\mathrm{pt}}\left(\left(3 m_{c}\right)^{2} ; n_{f}=3\right)=0.070502=0.221487 / \pi$ [Eq. 24)] at the scale $Q^{2}=\left(3 m_{c}\right)^{2}$ to the high final scale $Q^{2}=M_{Z}^{2}$ gives the value $a_{\mathrm{pt}}\left(M_{Z}^{2} ; n_{f}=3\right)=0.033694=0.105852 / \pi$. The analytic coupling, for both representative values of parameter $s_{0}$ used later in this work $\left(s_{0}=3.858,6.862\right)$, gives the same value $0.221487 / \pi$ at $Q^{2}=\left(3 m_{c}\right)^{2}$, and almost the same values at $Q^{2}=M_{Z}^{2}: \mathcal{A}_{1}\left(M_{Z}^{2}\right)=0.105853 / \pi, 0.105856 / \pi$, respectively. ${ }^{9}$ This strongly indicates that $\mathcal{A}_{1}\left(Q^{2}\right)$ of the analytic QCD model presented in this work, at both mentioned values of $s_{0}$, is practically indistinguishable from the perturbative $a_{\mathrm{pt}}\left(Q^{2}\right)$ at scales $Q^{2}>\left(3 m_{c}\right)^{2}$. This conclusion even gets generalized to any higher order couplings of this analytic QCD and the perturbative QCD [see the comments in the paragraph at Eq. [39]].

Altogether, the three conditions (21), 222, and 25) eliminate three of the four otherwise free parameters $s_{0}, s_{1}, f_{1}^{2}, \Lambda^{2}$ of our analytic QCD model. We are thus left with only one free parameter, e.g., the dimensionless parameter $s_{0}$ in Eq. 9 for $\mathcal{A}_{1}\left(Q^{2}\right)$. In Table I] we present the numerical dependence of the parameters $s_{1}, f_{1}^{2}$ on $s_{0}$. It turns out that the value of the scale $\Lambda \approx 0.487 \mathrm{GeV}$ is practically independent of the value of $s_{0}$, it varies by less than $0.1 \%$ for the range of the $s_{0}$-values presented in Table If the reason being that $\mathcal{A}_{1}\left(\left(3 m_{c}\right)^{2}\right)=a_{\mathrm{pt}}\left(\left(3 m_{c}\right)^{2}\right)$ $(=0.07050)$ behaves at such scales practically as perturbative coupling $a_{\mathrm{pt}}$ due to "approximate perturbative QCD" conditions 21 -22. ${ }^{10}$ In the last two columns of Table I we include various evaluated contributions to the strangeless and massless semihadronic tau decay ratio $r_{\tau}$; these aspects will be discussed in the next section. ${ }^{11}$ In Table I we see

8 We use for the $\overline{\mathrm{MS}}$ beta function $\beta\left(a\left(Q^{2}\right)\right)$ at $Q^{2}=\left(3 m_{c}\right)^{2}$ and $n_{f}=3$ the Padé $[2 / 3]_{\beta}(a)$ based on the 4-loop polynomial $\overline{\mathrm{MS}}$ beta function; if using the latter (polynomial) form, we obtain slightly different value $a_{\text {in }}\left[\equiv a_{\mathrm{pt}}\left(\left(3 m_{c}\right)^{2} ; \beta_{2}=0, \beta_{3}=0, \cdots ; n_{f}=3\right)\right]$ $=0.07054$. It appears that, at such relatively large values of $a_{\mathrm{pt}}$, the Padé $[2 / 3]_{\beta}(a)$ is a better approximation to the full (yet unknown) $\overline{\mathrm{MS}}$ beta function.

9 The values of $A_{1}\left(Q^{2}\right)$, for various values of the parameter $s_{0}$, differ from the values of the perturbative coupling only at low $Q^{2}<$ $10 \mathrm{GeV}^{2}$; for example, the relative difference $a_{\mathrm{pt}}\left(Q^{2}\right) / \mathcal{A}_{1}\left(Q^{2}\right)-1$ is a monotonously decreasing function of $Q^{2}$ for $Q^{2}<10 \mathrm{GeV}^{2}$; in the interval $1 \mathrm{GeV}^{2}<Q^{2}<5 \mathrm{GeV}^{2}$ it falls from 0.02 to 0.0004 when $s_{0}=3.858$, and from 0.05 to 0.001 when $s_{0}=6.862$.

10 For example, for the two input values $s_{0}=3.858,6.862$ used later in the text, we have $\Lambda=0.48679,0.48687 \mathrm{GeV}$, respectively; the perturbative value of $\Lambda$ (at $n_{f}=3$ ) is $\Lambda_{p Q C D}=0.48676 \mathrm{GeV}$.

11 The value of $s_{0}=3.858$ results in the reproduction of the central value of the experimental result $r_{\tau}\left(\Delta S=0, m_{q}=0\right)_{\exp }=0.203 \pm 0.004$ when the leading- $\beta_{0}(\mathrm{LB})$ and the beyond-the-leading- $\beta_{0}(\mathrm{bLB})$ contributions are evaluated and added together. 
that the cutoff parameter $s_{0}=M_{0}^{2} / \Lambda^{2}$ cannot fall below $s_{0} \approx 1.96$ because in such a case $s_{1}=M_{1}^{2} / \Lambda^{2}$ turns out to be negative and the coupling acquires a Landau singularity (at $Q^{2}=-s_{1}>0$ ).

\section{EVALUATIONS OF INCLUSIVE LOW-ENERGY OBSERVABLES}

In this Section we present the results of evaluation of two inclusive low-energy observables in our discussed model. We recall that the high-energy observables (with $\left|Q^{2}\right| \gtrsim 10^{1} \mathrm{GeV}^{2}$ ) are reproduced in the model because at such energies the coupling practically agrees with the perturbative coupling

$$
\left|\mathcal{A}_{1}\left(Q^{2}\right)-a_{\mathrm{pt}}\left(Q^{2}\right)\right| \lesssim\left(\Lambda^{2} /\left|Q^{2}\right|\right)^{3} \quad\left(\left|Q^{2}\right| \gg \Lambda^{2}\right) .
$$

The goal here is to fix the only free parameter $s_{0}$ of the model by requiring reproduction of the measured low energy QCD observables. The most precisely measured inclusive low energy QCD observable is the semihadronic tau decay ratio $R_{\tau}$, which is the ratio of $\Gamma\left(\tau^{-} \rightarrow \nu_{\tau}\right.$ hadrons $\left.(\gamma)\right)$ and $\Gamma\left(\tau^{-} \rightarrow \nu_{\tau} e^{-} \bar{\nu}_{e}(\gamma)\right)$. After removing the (well measured) strangeness-changing contribution, the color and Cabibbo-Kobayashi-Maskawa factors and the electroweak effects, as well as the chirality-violating higher-twist ("quark mass") contributions ${ }^{12}$ we obtain the experimental value

$$
r_{\tau}\left(\triangle S=0, m_{q}=0\right)_{\exp .}=0.203 \pm 0.004 .
$$

For details of this extraction we refer to Appendix B of Ref. [20] and references therein. The quantity $r_{\tau}$ of Eq. 227] is timelike, but it can be obtained from the spacelike massless Adler function $d_{\mathrm{Adl}}\left(Q^{2}\right)=a_{\mathrm{pt}}\left(Q^{2}\right)+\mathcal{O}\left(a_{\mathrm{pt}}^{2}\right)$ by contour integration 38 .

$$
r_{\tau}=\frac{1}{2 \pi} \int_{-\pi}^{+\pi} d \phi\left(1+e^{i \phi}\right)^{3}\left(1-e^{i \phi}\right) d_{\mathrm{Adl}}\left(Q^{2}=m_{\tau}^{2} e^{i \phi}\right)
$$

The perturbative expansion of $d_{\mathrm{Adl}}\left(Q^{2}\right)$

$$
d_{\mathrm{Adl}}\left(Q^{2}\right)_{\mathrm{pt}}=a_{\mathrm{pt}}+\sum_{n=1}^{\infty}\left(d_{\mathrm{Adl}}\right)_{n} a_{\mathrm{pt}}^{n+1} .
$$

has been calculated up to $\mathcal{O}\left(a_{\mathrm{pt}}^{4}\right)$ 39 41, i.e., the coefficients $\left(d_{\mathrm{Adl}}\right)_{n}$ are known for $n=1,2,3$. Here, $a_{\mathrm{pt}}=$ $a_{\mathrm{pt}}\left(\mu^{2} ; c_{2}, c_{3}, \ldots\right)$ is at a chosen renormalization scale $\mu^{2}$ and renormalization scheme $\left(c_{2}, c_{3}, \ldots\right)$, where $c_{j}=\beta_{j} / \beta_{0}$ $(j \geq 2)$ are the renormalization scheme parameters. For our purposes it is more convenient to reorganize the expansion 290 in terms of the logarithmic derivatives

$$
\widetilde{a}_{\mathrm{pt}, n+1}\left(\mu^{2}\right) \equiv \frac{(-1)^{n}}{\beta_{0}^{n} n !} \frac{\partial^{n} a_{\mathrm{pt}}\left(\mu^{2}\right)}{\partial\left(\ln \mu^{2}\right)^{n}}=a_{\mathrm{pt}}^{n}+\mathcal{O}\left(a_{\mathrm{pt}}^{n+1}\right) \quad(n=1,2, \ldots),
$$

resulting in "modified perturbation" (mpt) series

$$
d_{\mathrm{Adl}}\left(Q^{2}\right)_{\mathrm{mpt}}=a_{\mathrm{pt}}+\sum_{n=1}^{\infty}\left(\widetilde{d}_{\mathrm{Adl}}\right)_{n} \widetilde{a}_{\mathrm{pt}, n+1}
$$

The first three coefficients $\left(\widetilde{d}_{\mathrm{Adl}}\right)_{n}(n=1,2,3)$ are known since they can be expressed via $\left(d_{\mathrm{Adl}}\right)_{k}$ 's $(k=1, \ldots, n)$. The basic idea of evaluation of such leading-twist expressions in general analytic QCD models is to replace (cf. Refs. [12, 13]; for MA, see also Ref. [15])

$$
a_{\mathrm{pt}} \mapsto \mathcal{A}_{1}, \quad \widetilde{a}_{\mathrm{pt}, n+1} \mapsto \widetilde{\mathcal{A}}_{n+1} \quad(n=1,2, \ldots) .
$$

where $\widetilde{\mathcal{A}}_{n+1}$ are the corresponding logarithmic derivatives in analytic QCD

$$
\widetilde{\mathcal{A}}_{n+1}\left(\mu^{2}\right)=\frac{(-1)^{n}}{\beta_{0}^{n} n !} \frac{\partial^{n} \mathcal{A}_{1}\left(\mu^{2}\right)}{\partial\left(\ln \mu^{2}\right)^{n}}, \quad(n=1,2, \ldots) .
$$

\footnotetext{
12 Further, assuming that the chirality-conserving higher twist effects are negligible, i.e., that the gluon condensate $\langle a G G\rangle$ is approximately zero.
} 
Therefore, the "modified perturbation" (mpt) series of perturbative QCD (31) is replaced in analytic QCD models by "modified analytic" (man) series

$$
d_{\mathrm{Adl}}\left(Q^{2}\right)_{\operatorname{man}}=\mathcal{A}_{1}+\sum_{n=1}^{\infty}\left(\widetilde{d}_{\mathrm{Adl}}\right)_{n} \widetilde{\mathcal{A}}_{n+1} .
$$

The known truncated series of Adler function in analytic QCD models is then

$$
d_{\mathrm{Adl}}\left(Q^{2}\right)_{\mathrm{man}}^{[N]}=\mathcal{A}_{1}+\left(\widetilde{d}_{\mathrm{Adl}}\right)_{1} \widetilde{\mathcal{A}}_{2}+\cdots\left(\widetilde{d}_{\mathrm{Adl}}\right)_{N-1} \widetilde{\mathcal{A}}_{N}
$$

with $N=4$, and with the coouplings $\mathcal{A}_{1}, \widetilde{\mathcal{A}}_{2}, \ldots$ at renormalization scale

$$
\mu^{2}=Q^{2} \exp (\mathcal{C}) \quad(\mathcal{C} \sim 1)
$$

This series is then inserted into the contour integral (28), resulting in the sum

$$
r_{\tau}^{(\mathrm{LO}+\mathrm{bLO})[\mathrm{N}]}=I\left(\mathcal{A}_{1}, \mathcal{C}\right)+\sum_{n=1}^{N-1}\left(\widetilde{d}_{\mathrm{Adl}}\right)_{n} I\left(\widetilde{\mathcal{A}}_{n+1}, \mathcal{C}\right),
$$

where $N=4$, and $I\left(\widetilde{\mathcal{A}}_{n+1}, \mathcal{C}\right)$ are the corresponding contour integrals of $\mathcal{A}_{1} \equiv \widetilde{\mathcal{A}}_{1}, \widetilde{\mathcal{A}}_{2}, \ldots$

$$
I\left(\widetilde{\mathcal{A}}_{n+1}, \mathcal{C}\right)=\frac{1}{2 \pi} \int_{-\pi}^{+\pi} d \phi\left(1+e^{i \phi}\right)^{3}\left(1-e^{i \phi}\right) \widetilde{\mathcal{A}}_{n+1}\left(e^{\mathcal{C}} m_{\tau}^{2} e^{i \phi}\right),
$$

The superscript "LO+bLO" in Eq. (37) indicates that this is a sum of the leading order (LO) term $I\left(\mathcal{A}_{1}, \mathcal{C}\right)$ and of the higher order terms beyond-the-leading-order (bLO).

The expansions 31$), 34-(35)$ are expansions in nonpower quantities $\widetilde{a}_{\mathrm{pt}, n+1}$ and $\widetilde{\mathcal{A}}_{n+1}$. The latter are constructed by applying logarithmic derivative operators directly on the couplings $a_{\mathrm{pt}}$ and $\mathcal{A}_{1}$, and are thus formally linear in the latter couplings. This is very convenient also for the application of linear integral transforms on the observables, since the linear integral transforms of $\mathcal{A}_{1}$ and $\widetilde{\mathcal{A}}_{n+1}$ usually become simply related and since these transforms respect the truncation of the series. In MA of Refs. [4, 5], the construction of the higher couplings also has such properties, and therefore, the transitions from momentum-transfer $\left(Q^{2}\right)$ picture to the energy $(s$ or $\sigma)$ picture and to the distance $(r)$ picture become elegant and transparent, especially since the truncation of such series is fully respected by the transformations (cf. Refs. [6, 35]).

In this context, we stress that the spacelike observables $\mathcal{D}\left(Q^{2}\right)$, such as Adler function or Bjorken polarized sum rule (BjPSR), at higher momentum-transfer scales $Q^{2}>\left(3 m_{c}\right)^{2}$ obtain practically the same value when evaluated in the perturbative QCD by the truncated (modified) perturbation series $d\left(Q^{2}\right)_{\mathrm{mpt}}^{[N]}[\mathrm{cf}$. Eq. $\sqrt[31]{31}]$ or evaluated in the presented analytic QCD model by the truncated (modified) analytic series $d\left(Q^{2}\right)_{\text {man }}^{[N]}$, Eq. 35$)$. This is so because:

- At such $Q^{2}$ the values of $a_{\mathrm{pt}}\left(Q^{2}\right)$ and $\mathcal{A}_{1}\left(Q^{2}\right)$ are practically equal, as a consequence of the fulfilled condition (17) with $k=3$ [see also the comments in the paragraph just after Eq. 25]].

- Applying logarithmic derivatives to the relation (17) with $k=3$, valid in the presented analytic QCD model, we conclude that

$$
\left|\widetilde{\mathcal{A}}_{n+1}\left(Q^{2}\right)-\widetilde{a}_{\mathrm{pt}, n+1}\left(Q^{2}\right)\right| \sim\left(\frac{\Lambda^{2}}{Q^{2}}\right)^{3} \quad\left(\left|Q^{2}\right| \gg \Lambda^{2} ; n=1,2, \ldots\right) .
$$

By analogy this implies that at $Q^{2}>\left(3 m_{c}\right)^{2}$ the values of $\widetilde{a}_{\mathrm{pt}, n+1}$ (in the renormalization scheme $\beta_{2}=\beta_{3}=\cdots=$ $0)$ and $\widetilde{\mathcal{A}}_{n+1}\left(Q^{2}\right)$ in the presented analytic QCD are practically equal. For example, if keeping $n_{f}=3$ fixed (in order not to worry about the threshold effects), we obtain numerically: $\widetilde{a}_{2}\left(\left(3 m_{c}\right)^{2}\right)=0.005593$ and $\widetilde{\mathcal{A}}_{2}\left(\left(3 m_{c}\right)^{2}\right)=$ $0.005592,0.005587$ (when $s_{0}=3.858,6.862$ ), and $\widetilde{a}_{2}\left(M_{Z}^{2}\right)=0.0012033$ and $\widetilde{\mathcal{A}}_{2}\left(M_{Z}^{2}\right)=0.0012033,0.0012034$ (when $\left.s_{0}=3.858,6.862\right)$; further, $\widetilde{a}_{3}\left(\left(3 m_{c}\right)^{2}\right)=0.000468$ and $\widetilde{\mathcal{A}}_{3}\left(\left(3 m_{c}\right)^{2}\right)=0.000467,0.000464$ (when $s_{0}=$ $3.858,6.862$ ), and $\widetilde{a}_{3}\left(M_{Z}^{2}\right)=0.0000442=\widetilde{\mathcal{A}}_{3}\left(M_{Z}^{2}\right)$ to the digits displayed (when $\left.s_{0}=3.858,6.862\right)$.

There is yet another, more sophisticated, way of evaluating inclusive spacelike and time-like observables. It is based on the knowledge of the leading- $\beta_{0}$ part of coefficients $d_{n}$ and $\widetilde{d}_{n}$ of the inclusive spacelike observable such as $d_{\mathrm{Adl}}\left(Q^{2}\right)$. These leading- $\beta_{0}$ (LB) parts can then be summed in any analytic QCD in the form

$$
\left(d_{\mathrm{Adl}}\right)_{\mathrm{an}}^{(\mathrm{LB})}\left(Q^{2}\right) \equiv \int_{0}^{\infty} \frac{d t}{t} F_{\mathrm{Adl}}^{\mathcal{E}}(t) \mathcal{A}_{1}\left(t Q^{2} e^{\overline{\mathcal{C}}}\right)
$$


where $\overline{\mathcal{C}}=-5 / 3$. The Euclidean $(\mathcal{E})$ characteristic function $F_{\text {Adl }}^{\mathcal{E}}(t)$ is known [42. Expansion of expression 400 in logarithmic derivatives $\widetilde{\mathcal{A}}_{n}\left(Q^{2}\right)$ then reproduces exactly the leading- $\beta_{0}$ part of the "modified analytic" expansion (34)

$$
\left(d_{\mathrm{Adl}}\right)_{\mathrm{an}}^{(\mathrm{LB})}\left(Q^{2}\right)=\mathcal{A}_{1}+\sum_{n=1}^{\infty} c_{n n}^{(1)} \beta_{0}^{n} \widetilde{\mathcal{A}}_{n+1} .
$$

where the expansion of each coefficient $\left(\widetilde{d}_{\mathrm{Adl}}\right)_{n}$ and $\left(d_{\mathrm{Adl}}\right)_{n}$ of the "mpt" and "pt" expansions, Eqs. 31] and 29, in powers of $\beta_{0}$ is

$$
\left(\widetilde{d}_{\mathrm{Adl}}\right)_{n}=\sum_{k=-1}^{n} \widetilde{c}_{n k}^{(1)} \beta_{0}^{k}, \quad\left(d_{\mathrm{Adl}}\right)_{n}=\sum_{k=-1}^{n} c_{n k}^{(1)} \beta_{0}^{k}, \quad \widetilde{c}_{n n}^{(1)}=c_{n n}^{(1)} .
$$

In practice, we know the full coefficients $\left(\widetilde{d}_{\mathrm{Adl}}\right)_{n}$ for $n=1,2,3$. Subtracting from them the leading- $\beta_{0}$ parts $c_{n n}^{(1)} \beta_{0}^{n}$ then gives us a truncated series for the beyond-the-leading- $\beta_{0}$ (bLB) contributions. Hence, the LB-resummed expression $(\mathrm{LB}+\mathrm{bLB})$ for the Adler function, in any analytic QCD model, is

$$
\left(d_{\mathrm{Adl}}\right)_{\operatorname{man}}^{(\mathrm{LB}+\mathrm{bLB})}\left(Q^{2}\right)^{[N]}=\int_{0}^{\infty} \frac{d t}{t} F_{\mathrm{Adl}}^{\mathcal{E}}(t) \mathcal{A}_{1}\left(t Q^{2} e^{\overline{\mathcal{C}}}\right)+\sum_{n=1}^{N-1}\left(T_{\mathrm{Adl}}\right)_{n} \widetilde{\mathcal{A}}_{n+1},
$$

where $N=4$ and

$$
\left(T_{\mathrm{Adl}}\right)_{n}=\left(\widetilde{d}_{\mathrm{Adl}}\right)_{n}-c_{n n}^{(1)} \beta_{0}^{n} .
$$

When inserting the expression 43 into the contour integral 28), we get the LB-resummed expression for $r_{\tau}$ in any analytic QCD model

$$
r_{\tau}^{(\mathrm{LB}+\mathrm{bLB}),[\mathrm{N}]}=r_{\tau}^{(\mathrm{LB})}+\sum_{n=1}^{N-1}\left(T_{\mathrm{Adl}}\right)_{n} I\left(\widetilde{\mathcal{A}}_{n+1}, \mathcal{C}\right),
$$

where $I\left(\widetilde{\mathcal{A}}_{n+1}, \mathcal{C}\right)$ are given in Eq. 38 , and the LB-part is obtained by using the LB-integral 40 in contour integral 28). In Ref. [4] this expression was expressed in terms of the Minkowskian coupling $\mathfrak{A}_{1}(s)$

$$
\mathfrak{A}_{1}(s)=\frac{1}{\pi} \int_{s}^{\infty} \frac{d \sigma}{\sigma} \rho_{1}(\sigma)
$$

in the form

$$
r_{\tau}^{(\mathrm{LB})}=\int_{0}^{\infty} \frac{d t}{t} F_{r}^{\mathcal{M}}(t) \mathfrak{A}_{1}\left(t e^{\mathcal{C}} m_{\tau}^{2}\right)
$$

where the Minkowskian characteristic function $F_{r}^{\mathcal{M}}(t)$ was calculated explicitly. ${ }^{13}$ Using the relation $d \mathfrak{A}_{1}(s) / d \ln s=$ $-\rho_{1}(s) / \pi$ and performing integration by parts, the LB-contribution to (the massless and strangeless) $r_{\tau}$ can be rewritten in the following form in terms of the discontinuity function $\rho_{1}(\sigma)$, which is more convenient for numerical evaluations:

$$
r_{\tau}^{(\mathrm{LB})}=\frac{1}{\pi} \int_{0}^{\infty} \frac{d t}{t} \widetilde{F}_{r}(t) \rho_{1}\left(t e^{\overline{\mathcal{C}}} m_{\tau}^{2}\right)
$$

where

$$
\widetilde{F}_{r}(t)=\int_{0}^{t} \frac{d t^{\prime}}{t^{\prime}} F_{r}^{\mathcal{M}}\left(t^{\prime}\right)
$$

This expression was used in Refs. [19, 20, and explicit expression for the characteristic function $\widetilde{F}_{r}(t)$ is given in Ref. 20] (Appendix D).

${ }^{13}$ Since we use a different normalization, $F_{r}^{\mathcal{M}}(t)$ here is equal to $(t / 4)$ times $F_{r}^{\mathcal{M}}(t)$ of Ref. 43 . 
For derivations of and more details on the above identities, we refer to Refs. [12, 13, 19, 20].

For the evaluations of the (strangeless and massless) $r_{\tau}$ in our model, we use the LB-resummed expression (45) and, as an alternative, the more rudimentary expression (37), both with $N=4$. In Table I] of the previous Section, we included the results of these evaluations in the last two columns. We used the renormalization scale parameter $\mathcal{C}=0$, i.e., $\left|\mu^{2}\right|=m_{\tau}^{2}$ on the contour integral [cf. Eqs. (36), [38)]. We note that the leading- $\beta_{0}$ contribution Eq. (48) [and: Eq. [40]] is independent of renormalization scale. From the results of Table I we see that, when the value of the model parameter $s_{0}=M_{0}^{2} / \Lambda^{2}$ increases, the value of $r_{\tau}$ slowly decreases in the LB-resummed approach (LB+bLB) Eq. (45), and slowly increases in the other $(\mathrm{LO}+\mathrm{bLO})$ approach Eq. (37). The approach of Eq. (45) reproduces the central experimental value (27) $r_{\tau}=0.203$ at the parameter value $s_{0}=3.858$; the approach of Eq. (37) reproduces $r_{\tau}=0.203$ at $s_{0}=6.862$.

The running couplings $\mathcal{A}_{1}\left(Q^{2}\right)$ and $\widetilde{\mathcal{A}}_{n}\left(Q^{2}\right)(n=2,3)$ for positive $Q^{2}\left(0 \leq Q^{2} \leq\left(3 m_{c}\right)^{2}\right)$ are depicted in Figs. 2 and 3 for the aforementioned choices of parameter values $\left(s_{0}=3.858\right.$ and 6.862$)$, respectively. In these Figures, the perturbative couplings (in the same $\beta_{2}=\beta_{3}=\cdots=0$ renormalization scheme) are also presented, for comparison. We note that the analytic coupling $\mathcal{A}_{1}\left(Q^{2}\right)$ coincides with the perturbative coupling $a_{\mathrm{pt}}\left(Q^{2}\right)$ at $Q^{2}=\left(3 m_{c}\right)^{2}$. The conditions (21)- 22 bring the behavior of the analytic couplings very close to those of perturbative couplings in the high momentum regime $\left|Q^{2}\right| \gg \Lambda^{2}$ (note: $\Lambda^{2} \approx 0.24 \mathrm{GeV}^{2}$ ). This is clearly seen in Figs. 2 and 3 where the perturbative couplings $a_{\mathrm{pt}}, \tilde{a}_{\mathrm{pt}, 2}$ and $\tilde{a}_{\mathrm{pt}, 3}$ (dotted curves) virtually agree with the corresponding analytic couplings $\mathcal{A}_{1}, \widetilde{\mathcal{A}}_{2}$ and $\widetilde{\mathcal{A}}_{3}$ for $Q^{2}$ down to $Q^{2} \approx 2 \mathrm{GeV}^{2}$. The Landau singularities of the perturbative couplings appear for $Q^{2} \leq Q_{b}^{2}$ where the branching point is $Q_{b}^{2}=c_{1}^{-c 1 / \beta_{0}} \Lambda^{2}\left(\approx 0.635 \Lambda^{2} \approx 0.1504 \mathrm{GeV}^{2}\right.$ when $\left.n_{f}=3\right)$, and perturbative couplings diverge at the branching point $Q^{2}=Q_{b}^{2}$ (Landau pole). The discontinuity function $\rho_{1}(\sigma)=\operatorname{Im} \mathcal{A}_{1}\left(Q^{2}=-\sigma-i \epsilon\right)$ for the
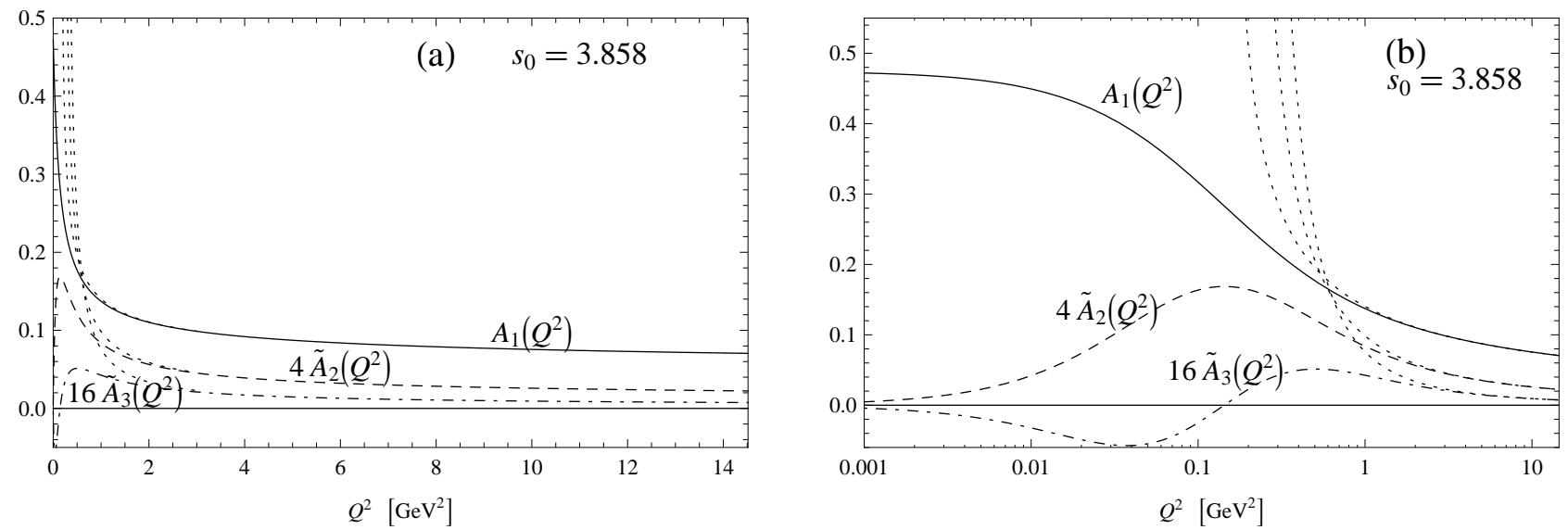

FIG. 2: The analytic couplings $\mathcal{A}_{1}\left(Q^{2}\right)$ (full curve), $\widetilde{\mathcal{A}}_{2}\left(Q^{2}\right)$ (dotted curve) and $\widetilde{\mathcal{A}}_{3}\left(Q^{2}\right)$ (dot-dashed curve) for positive $Q^{2}\left(0 \leq Q^{2} \leq\right.$ $\left.\left(3 m_{c}\right)^{2}\right)$ for the choice of parameter $s_{0}=3.858$ when: (a) linear scale is used for $Q^{2}$; (b) logarithmic scale is used for $Q^{2}$. For comparison, the corresponding perturbative couplings $a_{\mathrm{pt}}\left(Q^{2}\right), \tilde{a}_{\mathrm{pt}, 2}\left(Q^{2}\right)$ and $\tilde{a}_{\mathrm{pt}, 3}\left(Q^{2}\right)$ are included as dotted curves (in the renormalization scheme $\beta_{2}=\beta_{3}=\cdots=0$, with $\left.n_{f}=3\right)$. The couplings $\widetilde{\mathcal{A}}_{2}\left(Q^{2}\right)$ and $\tilde{a}_{\mathrm{pt}, 2}\left(Q^{2}\right)$ were rescaled by factor 4 , and the couplings $\widetilde{\mathcal{A}}_{3}\left(Q^{2}\right)$ and $\tilde{a}_{\mathrm{pt}, 3}\left(Q^{2}\right)$ by factor 16 , for better visibility.

two cases $s_{0}=3.858$ and 6.862 is presented in Figs. 1 (a), (b), respectively; it differs very much from the perturbative analog $\rho_{1}^{\mathrm{pt}}(\sigma)$ which is also included there.

A closer look at the two approaches, Eqs. 45) and (37), reveals:

- The simple approach Eq. (37) for $r_{\tau}$ in this model requires quite a large IR cut $M_{0}$ for the MA-discontinuity function $M_{0}=\Lambda \sqrt{s_{0}} \approx 1.28 \mathrm{GeV}$ which appears to be dangerously close to the mass of the process $m_{\tau}=1.777$ $\mathrm{GeV}$; in such a case the scales $|Q| \approx m_{\tau}$ are close to the energy regime $0<\sigma<M_{0}$ where the discontinuity function $\rho_{1}(\sigma)$ is parametrized by only one delta function - cf. Fig. 11(b). On the other hand, the LB-resummed approach Eq. 45 requires that the IR cutoff be $M_{0}=\Lambda \sqrt{s_{0}} \approx 0.96 \mathrm{GeV}$, roughly half of the mass $m_{\tau}-$ cf. Fig. 1(a).

- The convergence properties of the truncated "modified analytic" sum Eq. (37) for $r_{\tau}$ show that the last (fourth) term is appreciable $(\approx 0.017)$. On the other hand, the last (fourth) term in the sum Eq. 445 is significantly smaller $(\approx 0.005)$ - see Table $[\mathrm{I}$ in the case of the renormalization scale parameter $\mathcal{C}=0$.

- When varying the parameter $\mathcal{C}$, Eq. $(36)$, away from $\mathcal{C}=0$ upwards, e.g., in the interval between 0 and $\ln (2)$ (i.e., $\left|\mu^{2}\right|$ on the contour between $m_{\tau}^{2}$ and $2 m_{\tau}^{2}$ ), the result for $r_{\tau}$ in the approach of Eq. (37) decreases by several percent, while in the leading- $\beta_{0}$ resummed approach of Eq. 45) it remains virtually unchanged - 

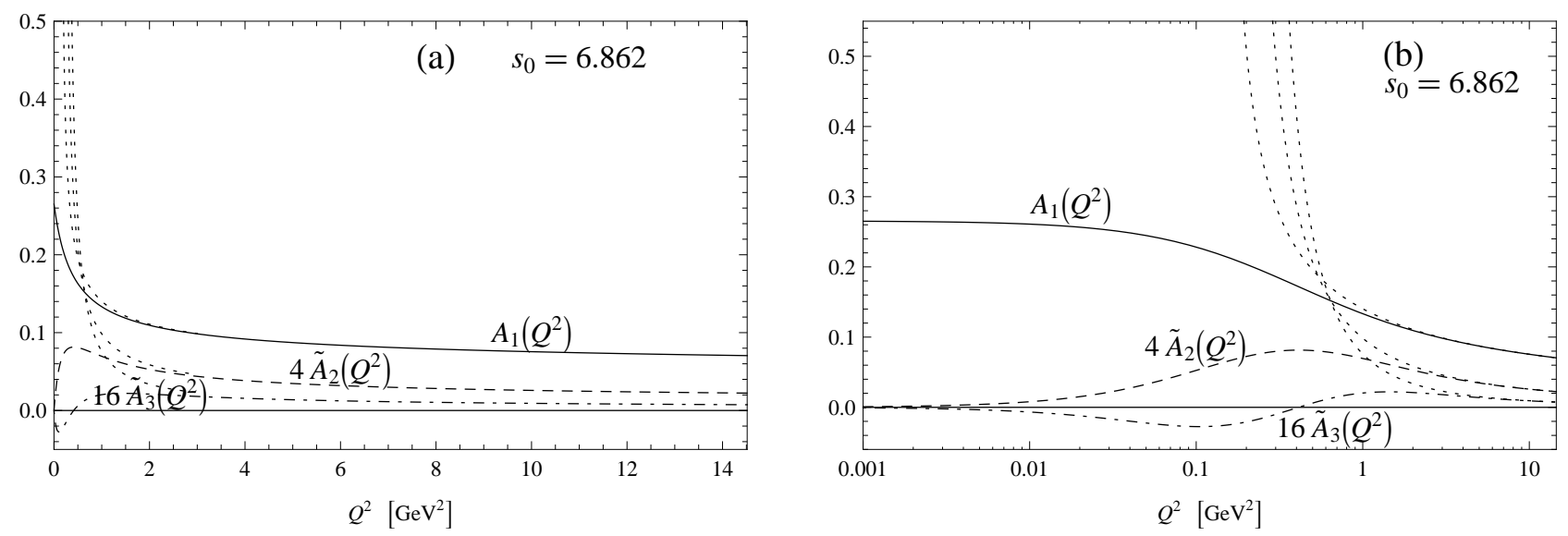

FIG. 3: Same as in Figs. 2 (a), (b), but now for the choice of the analytic QCD parameter $s_{0}=6.862$.

TABLE II: The four terms in truncated analytic expansions 45 and (37) for $r_{\tau}$, for the values of the $s_{0}$ parameter $s_{0}=3.858$ and $s_{0}=6.862$, respectively. The renormalization scale parameter $\mathcal{C}$ is varied from $\ln (3 / 4)$ to $\ln 2$. For $\mathcal{C}=0$, the two methods, with their respective values of $s_{0}$, reproduce the central experimental value $r_{\tau}=0.203$.

\begin{tabular}{ll|lllll}
\hline \hline method, $s_{0}$ & $\mathcal{C}$ & $r_{\tau}: \mathrm{LB}(\mathrm{LO})$ & $\mathrm{NLB}(\mathrm{NLO})$ & $\mathrm{N}^{2} \mathrm{LB}\left(\mathrm{N}^{2} \mathrm{LO}\right)$ & $\mathrm{N}^{3} \mathrm{LB}\left(\mathrm{N}^{3} \mathrm{LO}\right)$ & sum $(\mathrm{sum})$ \\
\hline \multirow{2}{*}{$\mathrm{LB}+\mathrm{bLB}}$, & $\ln (0.75)$ & 0.2156 & 0.0018 & -0.0258 & 0.0150 & 0.2068 \\
$s_{0}=3.858$ & 0 & 0.2156 & 0.0015 & -0.0190 & 0.0048 & 0.2030 \\
& $\ln (1.3)$ & 0.2156 & 0.0013 & -0.0148 & 0.0001 & 0.2022 \\
& $\ln 2$ & 0.2156 & 0.0011 & -0.0103 & -0.0032 & 0.2031 \\
LO+bLO, & $\ln (0.75)$ & 0.1458 & 0.0229 & 0.0238 & 0.0145 & 0.2070 \\
$s_{0}=6.862$ & $\ln (1.3)$ & 0.1323 & 0.0308 & 0.0224 & 0.0175 & 0.1970 \\
& $\ln 2$ & 0.1221 & 0.0354 & 0.0219 & 0.0164 & 0.1872 \\
\hline \hline
\end{tabular}

see Table II. When moving $\mathcal{C}$ to negative values $\left(\left|\mu^{2}\right|<m_{\tau}^{2}\right)$, the two approaches have mutually comparable stronger renormalization scale dependence, something to be expected since the model is apparently a simple approximation to the true situation for $\rho_{1}(\sigma)$ at low $\sigma<m_{\tau}^{2}$.

The dependence of these results on the renormalization scale $\mu^{2}$ is graphically presented in Fig. 4 . We see that the leading- $\beta_{0}$-resummed (i.e., LB+bLB) evaluation method of Eq. 45), in the depicted renormalization scale range $0.75 m_{\tau}^{2} \leq\left|\mu^{2}\right| \leq 2 m_{\tau}^{2}$, gives results for $r_{\tau}\left(\Delta S=0, m_{q}=0\right)$ that are significantly less renormalization scale dependent than those of the simpler evaluation method of Eq. (37).

We can compare these results with the corresponding results in perturbative QCD. We recall that the perturbative coupling $a_{\mathrm{pt}}\left(\mu^{2}\right)$ and our analytic coupling $\mathcal{A}_{1}\left(\mu^{2}\right)$ come together starting at renormalization scale $\mu^{2}=\left(3 m_{c}\right)^{2}$ upwards, and we have $a_{\mathrm{pt}}\left(\left(3 m_{c}\right)^{2}\right)=\mathcal{A}_{1}\left(\left(3 m_{c}\right)^{2}\right) \approx 0.07050$ in the renormalization scheme $\beta_{2}=\beta_{3}=\cdots=0$ [this value corresponds to the value $a\left(M_{Z}^{2}, \overline{\mathrm{MS}}\right) \approx 0.119 / \pi$, see Sec. II and to $\left.a\left(m_{\tau}^{2}, \beta_{2}=\beta_{3}=\cdots=0\right)=0.3065 / \pi\right]$. The perturbative QCD evaluation cannot use LB-resummation because Landau poles appear in the LB-integral. Therefore, we can compare only with the results of the $\mathrm{LO}+\mathrm{bLO}$ method in perturbative QCD (using the logarithmic derivatives $\widetilde{a}_{\mathrm{pt}, \mathrm{n}}$ 's in the contour integrals) and with the results of the usual perturbative "power" expansion method (using powers of $a_{\mathrm{pt}}$ in the contour integral). The results for $r_{\tau}$ are given in Table III for the two aforementioned methods in our analytic QCD model, and for the LO+bLO and "power" expansion method in perturbative QCD, at various values of the renormalization scale parameters $\mathcal{C}$. We see from Table III that the variation $\left|\Delta r_{\tau}\right|$ when the renormalization scale parameter $\mathcal{C}$ varies between $\ln (0.75)$ and $\ln 2$ is for the four methods $46,198,84,103$, respectively. The facility of unambiguous LB-resummation, which is possible only in analytic QCD models, leads to reduced renormalization scale dependence of the result for $r_{\tau}$. On the other hand, in perturbative QCD approaches the use of the logarithmic derivatives (pQCD LO+bLO) has the tendency to reduce the renormalization scale dependence of the result for $r_{\tau}$ in comparison with the use of the power expansion, something already noted in Ref. $44 .{ }^{14}$ We also note that in

14 In Ref. 44, perturbative QCD evaluation of the contour integral of $r_{\tau}$ was performed in $\overline{\mathrm{MS}}$ renormalization scheme, and in that 


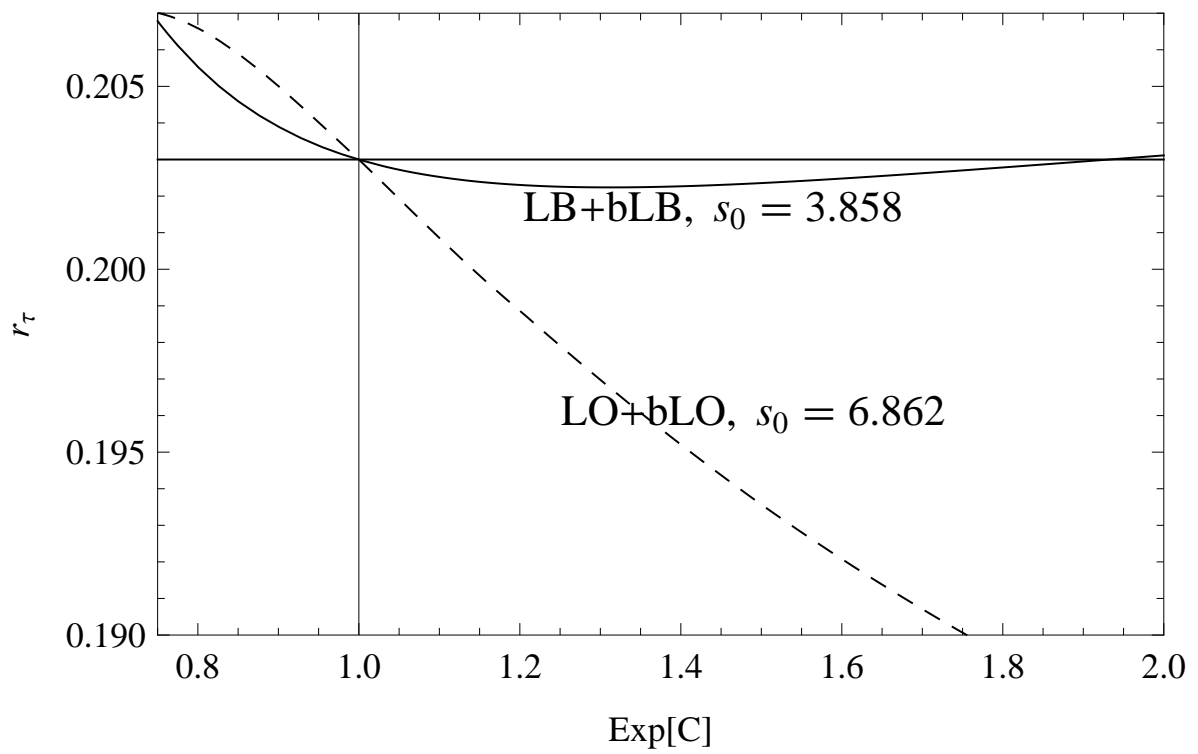

FIG. 4: The dependence of the evaluation method of Eq. 45) (LB+bLB) and of Eq. 37) (LO+bLO), for $r_{\tau}$, of the contour renormalization scale parameter $\exp (\mathcal{C}) \equiv\left|\mu^{2}\right| / m_{\tau}^{2}$. Values of the $s_{0}$ parameter $(3.858$ and 6.862 , respectively) were adjusted so that, at $\exp (\mathcal{C})=1$, the central experimental value $r_{\tau}\left(\Delta S=0, m_{q}=0\right)=0.203$ is reproduced.

TABLE III: Values of $r_{\tau}$ evaluated by the truncated analytic expansions Eq. (45) [LB+bLB] and Eq. (37) [LO+bLO] (for the values of the $s_{0}$ parameter $s_{0}=3.858$ and 6.862 , respectively), as well as values obtained by perturbative evaluations LO+bLO (involving $\widetilde{a}_{\mathrm{pt}, \mathrm{n}}$ 's in the contour integral) and the truncated "power" expansion (involving powers $a_{\mathrm{pt}}^{n}$ 's in the contour integral), in the renormalization scheme $\beta_{2}=\beta_{3}=\cdots=0$. At renormalization scale $\left(3 m_{c}\right)$, analytic QCD and perturbative QCD are assumed to merge: $a_{\mathrm{pt}}\left(\left(3 m_{c}\right)^{2}\right)=\mathcal{A}_{1}\left(\left(3 m_{c}\right)^{2}\right) \approx 0.07050$. The results are truncated sums of four terms. The renormalization scale parameter $\mathcal{C}$ is varied from $\ln (3 / 4)$ to $\ln 2$.

\begin{tabular}{l|ll|ll}
\hline \hline $\mathcal{C}$ & $r_{\tau}:$ LB+bLB & LO+bLO & pQCD LO+bLO & pQCD power exp. \\
\hline $\ln (0.75)$ & 0.2068 & 0.2070 & 0.1893 & 0.1856 \\
0 & 0.2030 & 0.2030 & 0.1873 & 0.1828 \\
$\ln (1.3)$ & 0.2022 & 0.1970 & 0.1850 & 0.1801 \\
$\ln 2$. & 0.2031 & 0.1872 & 0.1809 & 0.1753 \\
\hline \hline
\end{tabular}

perturbative QCD, in order to reproduce the correct value of $r_{\tau} \approx 0.203$, we need a larger value of $a_{\mathrm{pt}}$ that would correspond to $a_{\mathrm{pt}}\left(M_{Z}^{2}, \overline{\mathrm{MS}}\right) \approx 0.121 / \pi\left([44)\right.$; in our presented cases, we have $a_{\mathrm{pt}}\left(M_{Z}^{2}, \overline{\mathrm{MS}}\right) \approx 0.119 / \pi$, and therefore perturbative QCD gives too low a value of $r_{\tau} \approx 0.18-0.19$.

Another inclusive low energy QCD observable is, for example, Bjorken polarized sum rule $(\operatorname{BjPSR}) d_{\mathrm{Bj}}\left(Q^{2}\right)=$ $a\left(Q^{2}\right)+\mathcal{O}\left(a^{2}\right)$ at low $Q^{2}$. This is a spacelike quantity whose experimental values are included in the last two lines of Table IV for three representative values of $Q^{2}: 1.01,1.71$ and $2.92 \mathrm{GeV}^{2}$. These experimental data are based on the JLab CLAS EG1b (2006) measurements [45] of the $\Gamma_{1}^{\mathrm{p}-\mathrm{n}}\left(Q^{2}\right)$ sum rule for spin-dependent proton and neutron structure functions $g_{1}^{\mathrm{p}, \mathrm{n}}$ [46]. The measured quantity $\Gamma_{1}^{\mathrm{p}-\mathrm{n}}$ and the "canonical" BjPRS quantity $d_{\mathrm{Bj}}$ are related with each other in the following way:

$$
\begin{aligned}
\Gamma_{1}^{\mathrm{p}-\mathrm{n}}\left(Q^{2}\right) & \equiv \int_{0}^{1} d x_{\mathrm{Bj}}\left(g_{1}^{\mathrm{p}}\left(x_{\mathrm{Bj}}, Q^{2}\right)-g_{1}^{\mathrm{n}}\left(x_{\mathrm{Bj}}, Q^{2}\right)\right) \\
& =\frac{g_{A}}{6}\left(1-d_{\mathrm{Bj}}\left(Q^{2}\right)\right)+\sum_{j=2}^{\infty} \frac{\mu_{2 j}^{\mathrm{p}-\mathrm{n}}\left(Q^{2}\right)}{\left(Q^{2}\right)^{j-1}},
\end{aligned}
$$

where $g_{A}=1.267 \pm 0.004$ [34] is the triplet axial charge, $\left(1-d_{\mathrm{Bj}}\right)$ is the nonsinglet leading-twist Wilson coefficient,

scheme the difference between the renormalization scale dependence of the two approaches LO+bLO (there named: modified CIPT) and the power expansion approach (CIPT) was found to be even greater than in the here presented case of the renormalization scheme $\beta_{2}=\beta_{3}=\cdots=0$. 
TABLE IV: Bjorken polarized sum rule (BjPSR) results $d_{\mathrm{Bj}}\left(Q^{2}\right)$ evaluated with the truncated "modified analytic expansions" Eq. (43) $\left[\mathrm{LB}+\mathrm{bLB}\right.$, with $\left.s_{0}=3.858\right]$ and Eq. 35 [LO+bLO, with $\left.s_{0}=3.858,6.862\right]$, for $N=4$ and $N=3$. The renormalization scale parameter is taken $\mathcal{C}=0\left(\mu^{2}=Q^{2} \exp (\mathcal{C})\right)$. The number of active quark flavors is $n_{f}=3$. In brackets the variation of the result is given when the parameter $\mathcal{C}$ increases from zero to $\ln 2$, and when the renormalization scheme changes $($ at $\mathcal{C}=0$ ) from $\beta_{2}=\beta_{3}=\cdots=0$ to $\overline{\mathrm{MS}}$, respectively. The experimentally measured values are given in the last two lines (see the text for details).

\begin{tabular}{ll|lll}
\hline \hline$s_{0}$ & method & $d_{\mathrm{Bj}}\left(Q^{2}\right): Q^{2}=1.01 \mathrm{GeV}^{2}$ & $Q^{2}=1.71 \mathrm{GeV}^{2}$ & $Q^{2}=2.92 \mathrm{GeV}^{2}$ \\
\hline \multirow{2}{*}{3.858} & LB+bLB, $N=4$ & $0.1973[+2.1 \%,-10.0 \%]$ & $0.1755[+7.3 \%,-7.8 \%]$ & $0.1595[+7.2 \%,-4.3 \%]$ \\
& LB+bLB, $N=3$ & $0.2290[+6.1 \%,-5.3 \%]$ & $0.2066[+5.5 \%,-3.1 \%]$ & $0.1827[+4.3 \%,-0.8 \%]$ \\
\hline \multirow{2}{*}{3.858} & LO+bLO, $N=4$ & $0.2774[-1.3 \%,-1.3 \%]$ & $0.2234[-4.2 \%,-0.6 \%]$ & $0.1779[-4.6 \%,+0.0 \%]$ \\
& LO+bLO, $N=3$ & $0.2597[-8.7 \%,+1.5 \%]$ & $0.2061[-8.7 \%,+2.9 \%]$ & $0.1650[-7.5 \%,+3.3 \%]$ \\
\hline \multirow{2}{*}{6.862} & LO+bLO, $N=4$ & $0.2103[+9.1 \%,-0.7 \%]$ & $0.1926[+3.0 \%,-1.1 \%]$ & $0.1668[-1.0 \%,-0.7 \%]$ \\
& LO+bLO, $N=3$ & $0.2184[+0.0 \%,-2.2 \%]$ & $0.1898[-4.0 \%,-0.5 \%]$ & $0.1598[-5.5 \%,+1.1 \%]$ \\
\hline \multirow{2}{*}{} & exp. & $0.23 \pm 0.18$ & $0.13 \pm 0.12$ & $0.09 \pm 0.07$ \\
& & $0.23 \pm 0.12 \pm 0.13$ & $0.13 \pm 0.09 \pm 0.08$ & $0.09 \pm 0.05 \pm 0.05$ \\
\hline \hline
\end{tabular}

while $\mu_{2 j}^{\mathrm{p}-\mathrm{n}} / Q^{2 j-2}(j \geq 2)$ are the higher-twist contributions. The measured JLab values of $\Gamma_{1}^{\mathrm{p}-\mathrm{n}}\left(Q^{2}\right)$, with the elastic contribution excluded, are [45]: $0.1236 \pm 0.0254$ for $Q^{2}=1.01 \mathrm{GeV}^{2} ; 0.1605 \pm 0.0195$ for $Q^{2}=1.71 \mathrm{GeV}^{2}$; $0.1789 \pm 0.0112$ for $Q^{2}=2.92 \mathrm{GeV}^{2}$. The values given in the last two lines of Table IV are obtained from these values by subtracting from the aforementioned measured values the first higher-twist term $\mu_{4}^{\mathrm{p}-\mathrm{n}} / Q^{2}$ with the value $\mu_{4}^{\mathrm{p}-\mathrm{n}} \approx \mu_{4}^{\mathrm{p}-\mathrm{n}}(Q=1 \mathrm{GeV})=-0.040 \pm 0.028$ obtained by a three-parameter perturbative QCD fit in Ref. [45]. The central value $(-0.040)$ was reconfirmed in Refs. [4, 48, by a fit using the MA [4, 5, approach. In addition, in Ref. [47] it was shown, with the perturbative QCD and MA approach, that the the exclusion of the elastic contribution leads to strongly suppressed coefficients $\mu_{4}^{\mathrm{p}-\mathrm{n}}$ at the higher-twist terms $\sim 1 /\left(Q^{2}\right)^{j-1}$ with $j \geq 3$. In the second line of experimental values in Table IV] the uncertainties were split into the contribution coming from the uncertainty of the measured value of $\Gamma_{1}^{\mathrm{p}-\mathrm{n}}$ and the one from the uncertainty of the fitted value $\mu_{4}^{\mathrm{p}-\mathrm{n}}$.

The first two coefficients $d_{1}$ and $d_{2}$ in the expansion of $d_{\mathrm{Bj}}$ were obtained in Refs. 49] (given there in $\overline{\mathrm{MS}}$ renormalization scheme); the third coefficient $d_{3}$ is not known exactly, but estimates are known, e.g., Ref. [50]): in $\overline{\mathrm{MS}}$ scheme and at renormalization scale $\mu^{2}=Q^{2}$, it is $\left(\bar{d}_{\mathrm{Bj}}\right)_{3}=130$; we will use this value. The characteristic function $F_{\mathcal{D}}(t)$ for the leading- $\beta_{0}$ resummation for BjPSR was calculated and used in Ref. 12] (on the basis of the known [51] leading- $\beta_{0}$ parts of coefficients), and was presented in Ref. [13. This allows us to apply the evaluation methods of Eq. (43) and Eq. (35) in our analytic QCD model (in $\beta_{2}=\beta_{3}=\cdots=0$ renormalization scheme) for $d_{\mathrm{Bj}}\left(Q^{2}\right)$. The results of these two methods, at three different low squared momenta $Q^{2}\left(Q^{2}=1.01,1.71,2.92 \mathrm{GeV}^{2}\right)$ are presented in Table IV. We present in Table IV the results both in the case when the $\mathrm{N}^{3} \mathrm{LB}$ (and $\mathrm{N}^{3} \mathrm{LO}$ ) terms of $\sim \mathcal{A}_{4}$ are included in $d_{\mathrm{Bj}}\left(Q^{2}\right)$ ( $N=4$ case), and when they are not included $(N=3$ case). Further, variations of the results under the change of renormalization scale and scheme are also given. The renormalization scale was varied from the original $\mu^{2}=Q^{2}$ $(\mathcal{C}=0)$ to $\mu^{2}=2 Q^{2}(\mathcal{C}=\ln 2)$. The renormalization scheme was varied from the original scheme $\beta_{2}=\beta_{3}=\cdots=0$ to the $\overline{\mathrm{MS}}$ scheme $\beta_{2}=10.0599$ and $\beta_{3}=47.2281\left(n_{f}=3\right)$.

The change of the renormalization scheme was performed in the following way (cf. Ref. [13]). The dependence of couplings on the renormalization scheme parameters $c_{2} \equiv \beta_{2} / \beta_{0}$ and $c_{3} \equiv \beta_{3} / \beta_{0}$ is governed by the partial differential equations (pDFs) that are obtained from the corresponding pDFs of perturbative QCD under the analytization rule 32

$$
\begin{aligned}
& \frac{\partial \mathcal{A}_{1}\left(Q^{2} ; c_{2}, c_{3}\right)}{\partial c_{2}}=\frac{1}{2} \frac{\partial^{2} \mathcal{A}_{1}}{\partial x^{2}}+\frac{5}{12} c_{1} \frac{\partial^{3} \mathcal{A}_{1}}{\partial x^{3}}, \\
& \frac{\partial \widetilde{\mathcal{A}}_{2}\left(Q^{2} ; c_{2}, c_{3}\right)}{\partial c_{2}}=\frac{1}{2} \frac{\partial^{2} \widetilde{\mathcal{A}}_{2}}{\partial x^{2}}, \\
& \frac{\partial \mathcal{A}_{1}\left(Q^{2} ; c_{2}, c_{3}\right)}{\partial c_{3}}=-\frac{1}{12} \frac{\partial^{3} \mathcal{A}_{1}}{\partial x^{3}},
\end{aligned}
$$

where $x \equiv \beta_{0} \ln \left(Q^{2} / \Lambda^{2}\right)$. The left-hand sides of these pDFs are truncated, i.e., terms of $\sim \mathcal{A}_{5}\left(\sim A_{1}^{5}\right)$ are ignored, because the truncated series for $d_{\mathrm{Bj}}\left(Q^{2}\right)$ is known only up to $\sim \mathcal{A}_{4}$ (if the aforementioned estimated value of $\left(d_{\mathrm{Bj}}\right)_{3}$ is used).

The results in Table IV show that the values of $d_{\mathrm{Bj}}\left(Q^{2}\right)$ obtained with the (LB+bLB) method of Eq. (43) with $N=4$ are worse than those obtained with $N=3$, since the renormalization scale and scheme dependence is in general stronger in the $N=4$ case. This has to do with the numerical behavior of the $d_{\mathrm{Bj}}\left(Q^{2}\right)$ series in the approach 
TABLE V: The coefficients $\left(\widetilde{d}_{\mathrm{Adl}}\right)_{n}$ and $\left(\widetilde{d}_{\mathrm{Bj}}\right)_{n}$ for $n=1,2,3$, and their leading- $\beta_{0}(\mathrm{LB})$ counterparts, in two renormalization schemes: $\beta_{2}=\beta_{3}=\cdots=0$, and in $\overline{\mathrm{MS}}$ renormalization scheme. The renormalization scale parameter is $\mathcal{C}=0$, and $n_{f}=3$.

\begin{tabular}{|c|c|c|c|c|c|c|c|}
\hline Quantity & RSch & $\widetilde{d}_{1}$ & $\tilde{d}_{1}^{(\mathrm{LB})}$ & $\widetilde{d}_{2}$ & $\tilde{d}_{2}^{(\mathrm{LB})}$ & $\widetilde{d}_{3}$ & $\tilde{d}_{3}^{(\mathrm{LB})}$ \\
\hline \multirow{2}{*}{ Adler } & $\beta_{2}=\beta_{3}=\cdots=0$ & 1.640 & 1.556 & 7.93 & 15.71 & 39.00 & 24.83 \\
\hline & $\overline{\mathrm{MS}}$ & 1.640 & 1.556 & 3.46 & 15.71 & 26.38 & 24.83 \\
\hline \multirow{2}{*}{ BjPSR } & $\beta_{2}=\cdots=0$ & 3.583 & 4.5 & 18.32 & 32.34 & 91.13 & 255.23 \\
\hline & $\overline{\mathrm{MS}}$ & 3.583 & 4.5 & 13.84 & 32.34 & 52.45 & 255.23 \\
\hline
\end{tabular}

TABLE VI: The four terms in truncated analytic expansions 35 of $d_{\mathrm{Bj}}\left(Q^{2}\right)$ in our analytic QCD model with $s_{0}=6.862,3.858$; and the terms in the corresponding perturbative expansion in $\widetilde{a}_{\mathrm{pt}, n}$ 's ("pQCD LO+bLO"); and the terms in the usual power expansion, for $Q^{2}=1.01,1.71$ and $2.92 \mathrm{GeV}^{2}$. The renormalization scale is chosen as $\mu^{2}=Q^{2}$; renormalization scheme is: $\beta_{2}=\beta_{3}=\cdots=0$; in parentheses, the corresponding values in $\overline{\mathrm{MS}}$ renormalization scheme are given.

\begin{tabular}{|c|c|c|c|c|c|c|}
\hline$Q^{2}\left[\mathrm{GeV}^{2}\right]$ & method & $d_{\mathrm{Bj}}\left(Q^{2}\right): \mathrm{LO}$ & $\mathrm{NLO}$ & $\mathrm{N}^{2} \mathrm{LO}$ & $\mathrm{N}^{3} \mathrm{LO}$ & sum \\
\hline \multirow{4}{*}{1.01} & $\mathrm{LO}+\mathrm{bLO}\left(s_{0}=6.862\right)$ & $0.1329(0.1384)$ & $0.0621(0.0575)$ & $0.0234(0.0177)$ & $-0.0081(-0.0047)$ & $0.2103(0.2089)$ \\
\hline & $\mathrm{LO}+\mathrm{bLO}\left(s_{0}=3.858\right)$ & $0.1367(0.1471)$ & $0.0746(0.0798)$ & $0.0485(0.0366)$ & $0.0176(0.0102)$ & $0.2774(0.2738)$ \\
\hline & pQCD LO+bLO & $0.1398(0.1603)$ & $0.0874(0.1368)$ & $0.0857(0.1587)$ & $0.0861(0.2071)$ & $0.3989(0.6628)$ \\
\hline & pQCD power exp. & $0.1398(0.1603)$ & $0.0700(0.0920)$ & $0.0674(0.0832)$ & $0.0658(0.0858)$ & $0.3430(0.4213)$ \\
\hline \multirow{4}{*}{1.71} & $\mathrm{LO}+\mathrm{bLO}\left(s_{0}=6.862\right)$ & $0.1142(0.1198)$ & $0.0507(0.0502)$ & $0.0249(0.0188)$ & $0.0028(0.0016)$ & $0.1926(0.1905)$ \\
\hline & $\mathrm{LO}+\mathrm{bLO}\left(s_{0}=3.858\right)$ & $0.1154(0.1229)$ & $0.0551(0.0622)$ & $0.0356(0.0269)$ & $0.0173(0.0099)$ & $0.2234(0.2219)$ \\
\hline & pQCD LO+bLO & $0.1162(0.1266)$ & $0.0584(0.0769)$ & $0.0454(0.0596)$ & $0.0360(0.0506)$ & $0.2559(0.3136)$ \\
\hline & pQCD power exp. & $0.1162(0.1266)$ & $0.0484(0.0574)$ & $0.0387(0.0410)$ & $0.0314(0.0333)$ & $0.2347(0.2583)$ \\
\hline \multirow{4}{*}{2.92} & $\mathrm{LO}+\mathrm{bLO}\left(s_{0}=6.862\right)$ & $0.0991(0.1040)$ & $0.0397(0.0418)$ & $0.0209(0.0158)$ & $0.0070(0.0040)$ & $0.1668(0.1656)$ \\
\hline & $\mathrm{LO}+\mathrm{bLO}\left(s_{0}=3.858\right)$ & $0.0994(0.1045)$ & $0.0411(0.0474)$ & $0.0245(0.0186)$ & $0.0128(0.0074)$ & $0.1779(0.1779)$ \\
\hline & pQCD LO+bLO & $0.0996(0.1057)$ & $0.0418(0.0505)$ & $0.0269(0.0298)$ & $0.0176(0.0188)$ & $0.1860(0.2047)$ \\
\hline & pQCD power exp. & $0.0996(0.1057)$ & $0.0355(0.0400)$ & $0.0244(0.0239)$ & $0.0170(0.0162)$ & $0.1765(0.1857)$ \\
\hline
\end{tabular}

of Eq. 43 in this model, because the fourth term $\left(\mathrm{N}^{3} \mathrm{LB}, \sim \widetilde{\mathcal{A}}_{4}\right)$ is comparable or even larger than the third term $\left(\mathrm{N}^{2} \mathrm{LB}, \sim \widetilde{\mathcal{A}}_{3}\right)$ in this approach. E.g., for $Q^{2}=1.71 \mathrm{GeV}^{2}$, the series is (when $\mathcal{C}=0$ and renormalization scheme $\left.\beta_{2}=\cdots=0\right): d_{\mathrm{Bj}}\left(Q^{2}\right) \approx 0.248-0.014-0.027-0.031-\cdots$. We conclude that the leading- $\beta_{0}$ resummed approach of Eq. (43) is not working well for $d_{\mathrm{Bj}}\left(Q^{2}\right)$ at low $Q^{2}$, i.e., that the leading- $\beta_{0}$ terms are numerically not representative (of at least some) of the perturbative coefficients $\left(\widetilde{d}_{\mathrm{Bj}}\right)_{n}(n=2,3, \ldots)$. The latter fact is shown in Table $\mathrm{V}$. In that Table, the perturbative coefficients $\left(\widetilde{d}_{\mathrm{Adl}}\right)_{n}$ and their leading- $\beta_{0}$ (LB) counterparts are also given; these coefficients were relevant in the evaluation of $r_{\tau}$. Note that the leading- $\beta_{0}$ coefficients are renormalization scheme independent, they depend only on the renormalization scale. Comparing the coefficients $\left(\widetilde{d}_{\mathrm{Bj}}\right)_{3}$ and $\left(\widetilde{d}_{\mathrm{Adl}}\right)_{3}$ with their leading- $\beta_{0}$ counterparts, we can understand why the approach of Eq. 43 with $N=4$ is expected to work better in the evaluation of $r_{\tau}$ than in the evaluation of $d_{\mathrm{Bj}}\left(Q^{2}\right)$. We recall that the $\mathrm{N}^{3} \mathrm{LB}$ term in this evaluation of a spacelike observable $\mathcal{D}\left(Q^{2}\right)$ is $T_{3} \widetilde{\mathcal{A}}_{4}\left(e^{\mathcal{C}} Q^{2}\right)$ where $T_{3}=\left(\widetilde{d}_{4}-\widetilde{d}_{4}^{(\mathrm{LB})}\right)$, cf. Eqs. $43--44$.

On the other hand, Table IV] shows that the simpler approach of Eq. (35) (LO+bLO), in our model gives results for $d_{\mathrm{Bj}}\left(Q^{2}\right)$ that in general get more stable under the renormalization scale and scheme variations when the number of terms increases from $N=3$ to $N=4$, for $Q^{2} \geq 1.7 \mathrm{GeV}^{2}$. The case of very low scale $Q^{2}=1.01 \mathrm{GeV}^{2}$ is an exception, and probably has to do with the fact that our analytic QCD model is not very reliable at such low energies. We note that for $s_{0}=6.862$, where this approach is also used, the threshold masses are relatively high: $M_{1}=\sqrt{s_{1}} \Lambda=0.612$ $\mathrm{GeV}$ and $M_{0}=\sqrt{s_{0}} \Lambda=1.275 \mathrm{GeV}$.

However, in order to see whether we have any better convergence behavior in the evaluation of $d_{\mathrm{Bj}}\left(Q^{2}\right)$ than in the perturbative QCD, we should compare with the perturbative QCD evaluation of $d_{\mathrm{Bj}}\left(Q^{2}\right)$. The perturbative coupling and our analytic coupling merge starting at renormalization scale $\mu^{2}=\left(3 m_{c}\right)^{2}$ upwards, where we have $a_{\mathrm{pt}}\left(\left(3 m_{c}\right)^{2}\right)=\mathcal{A}_{1}\left(\left(3 m_{c}\right)^{2}\right) \approx 0.07050$, in the renormalization scheme $\beta_{2}=\beta_{3}=\cdots=0$ (this value corresponding to the value $a\left(M_{Z}^{2}, \overline{\mathrm{MS}}\right) \approx 0.119 / \pi$, see Sec. II . In Table VI we present the values of the evaluated expansion terms for $d_{\mathrm{Bj}}\left(Q^{2}\right)$ at various $Q^{2}$, in the approach of Eq. 35 in our model (i.e., in terms of $\widetilde{\mathcal{A}}_{n}$ 's), in the analogous approach in perturbative QCD (i.e., in terms of $\widetilde{a}_{\mathrm{pt}, n}$ 's), and in the usual power expansion approach in perturbative QCD (in powers of $a_{\mathrm{pt}}$ ), using the renormalization scale $\mu^{2}=Q^{2}$ (i.e., $\mathcal{C}=0$ ), all in the same renormalization scheme $\beta_{2}=\beta_{3}=\cdots=0$. In addition, the corresponding values in the $\overline{\mathrm{MS}}$ renormalization scheme are also given there (in parentheses). We see that the evaluated expansions in perturbative QCD for $d_{\mathrm{Bj}}\left(Q^{2}\right)$ at low momenta $Q^{2} \approx 1-2 \mathrm{GeV}^{2}$ 
behave much worse than the expansion Eq. (35) in our model; in fact, the third and the fourth terms are roughly the same in these perturbative expansions (in $\widetilde{a}_{\mathrm{pt}, n}$ 's; and in powers) in the renormalization scheme $\beta_{2}=\beta_{3}=\cdots=0$. Consequently, the expansions in perturbative QCD turn out to be very unreliable at such low values of $Q^{2}$. The situation in these perturbative QCD expansions becomes even worse in the $\overline{\mathrm{MS}}$ renormalization scheme; this has to do primarily with the fact that the offending Landau cut goes quite far into the positive regime in this scheme; the branching point (Landau pole) is at $Q_{b} \approx 0.388 \mathrm{GeV}$ and $0.627 \mathrm{GeV}$ in the two schemes, respectively.

The experimentally measured values are (Ref. 45]): $0.23 \pm 0.18$ for $Q^{2}=1.01 \mathrm{GeV}^{2} ; 0.13 \pm 0.12$ for $Q^{2}=1.71 \mathrm{GeV}^{2}$; $0.09 \pm 0.07$ for $Q^{2}=2.92 \mathrm{GeV}^{2}$; and are given also in Table IV Comparing the results of Tables IV and VI with these values, we see that the results of both methods, Eqs. (35) and (43), in the presented analytic QCD model, with $s_{0}=3.858$ and $s_{0}=6.862$, lie in general above the central experimental values, but in general within the large $1 \sigma$ uncertainties of the experimental values. On the other hand, the perturbative QCD results for $Q^{2}=1.01 \mathrm{GeV}^{2}$ and $Q^{2}=1.71 \mathrm{GeV}^{2}$ are significantly higher than those of the analytic QCD model. Some of the perturbative results lie outside the $1 \sigma$ interval of experimental values, and they show in general significantly worse convergence properties than the analytic QCD results. Furthermore, the experimental results indicate the tendency to lower values when $Q^{2}$ increases, and this is also the case of all the results in Table IV In the case of evaluation of Bjorken polarized sum rule in the present analytic QCD model, the method of Eq. (35), i.e., with no leading- $\beta_{0}$ resummation, should be regarded as the more reliable one. This is in contrast with the results for $r_{\tau}$ where we saw that the method of Eq. (45), which involves the leading- $\beta_{0}$ resummation, is in our analytic model more reliable and less renormalization scale dependent.

\section{CONCLUSIONS}

We presented a simple analytic QCD model which has initially three dimensionless parameters, and the scale parameter $\Lambda$. The model is obtained by parametrizing, in a specific renormalization scheme, the unknown behavior of the discontinuity function of the coupling in the IR regime by one delta function. The number of parameters in the model reduces from four to one by imposing the requirement that the analytic coupling $\mathcal{A}_{1}\left(Q^{2}\right)$ differ from the perturbative coupling $a_{\mathrm{pt}}\left(Q^{2}\right)$ at high $Q^{2}$ only by a small amount $\sim\left(\Lambda / Q^{2}\right)^{3}$. Therefore, the model merges with perturbative QCD at high energies $\left|Q^{2}\right|>10^{1} \mathrm{GeV}^{2}$ to a high degree of accuracy, and reproduces all the values of the high-energy QCD observables (with $\left|Q^{2}\right|>10^{1} \mathrm{GeV}^{2}$ ) just like the perturbative QCD does.

The remaining free dimensionless parameter $s_{0}$ is then adjusted so that the model reproduces the well measured strangeless semihadronic tau decay ratio $r_{\tau}$ - in the case when the leading- $\beta_{0}$ resummation is performed [Eq. (45); $s_{0}=3.858$ ] in the evaluation, and in the case when it is not performed [Eq. [37); $s_{0}=6.862$ ]. The evaluated results for the tau decay ratio in the approach of Eq. (45) turn out to be quite stable under the variation of the renormalization scale and show good convergence, not quite so the approach of Eq. (37). On the other hand, the evaluated values of the Bjorken polarized sum rule $d_{\mathrm{Bj}}\left(Q^{2}\right)$ at low momentum transfer $Q^{2}<3 \mathrm{GeV}^{2}$ in the presented model behave reasonably well under the variation of the renormalization scale and scheme, and show a reasonable good convergence, if no leading- $\beta_{0}$ resummation is performed; i.e., in the case of $d_{\mathrm{Bj}}\left(Q^{2}\right)$ the approach of Eq. (35) gives better results than the approach of Eq. 43 . The perturbative QCD evaluations of $d_{\mathrm{Bj}}\left(Q^{2}\right)$ at such low values of $Q^{2}$ turn out to be very unreliable.

It remains an outstanding problem how to perform in a numerically efficient way the change of the renormalization scheme for complex values of squared momentum transfer $Q^{2}$ in analytic QCD models in general, and in the presented model in particular. Solution of this problem would shed light on the degree of stability of the evaluated tau decay ratio $r_{\tau}$ under the renormalization scheme variation.

Another interesting problem would be to parametrize the unknown behavior of the discontinuity function of the coupling in the IR regime by two or more delta functions. This would allow us to fulfill the condition of merging the model with perturbative QCD (at $\left|Q^{2}\right|>10^{1} \mathrm{GeV}^{2}$ ) to an even higher degree of accuracy $\sim\left(\Lambda / Q^{2}\right)^{5}$, and would thus allow us to apply and interpret the Operator product expansion technique in such analytic QCD models in an analogous way as in perturbative QCD. 


\section{Acknowledgments}

This work was supported in part by FONDECYT (Chile) Grant No. 1095196 (C.C., G.C., O.E.), FONDECYT (Chile) Grant No. 1100348 (O.E.), Rings Project No. ACT119 (G.C.), and DAAD (H.E.M.).

[1] N.N. Bogoliubov and D.V. Shirkov, Introduction to the Theory of Quantum Fields, New York, Wiley, 1959 (first edition), 1980 (third edition).

[2] R. Alkofer, C. S. Fischer and F. J. Llanes-Estrada, Phys. Lett. B 611, 279 (2005) [Erratum-ibid. 670, 460 (2009)] arXiv:hep-th/0412330; A. C. Aguilar, D. Binosi and J. Papavassiliou, Phys. Rev. D 78, 025010 (2008) arXiv:0802.1870 hep-ph]]; A. C. Aguilar, D. Binosi, J. Papavassiliou and J. Rodriguez-Quintero, Phys. Rev. D 80, 085018 (2009) arXiv:0906.2633 [hep-ph]].

[3] A. Cucchieri and T. Mendes, Phys. Rev. Lett. 100, 241601 (2008) arXiv:0712.3517][hep-lat]]; I. L. Bogolubsky, E. M. Ilgenfritz, M. Muller-Preussker and A. Sternbeck, Phys. Lett. B 676, 69 (2009) arXiv:0901.0736 [hep-lat]].

[4] D. V. Shirkov and I. L. Solovtsov, hep-ph/9604363 Phys. Rev. Lett. 79, 1209 (1997) arXiv:hep-ph/9704333.

[5] K. A. Milton, I. L. Solovtsov and O. P. Solovtsova, Phys. Lett. B 415, 104 (1997) arXiv:hep-ph/9706409.

[6] D. V. Shirkov, Theor. Math. Phys. 127, 409 (2001) hep-ph/0012283; Eur. Phys. J. C 22, 331 (2001) hep-ph/0107282.

[7] A. V. Nesterenko, Phys. Rev. D 62, 094028 (2000); Phys. Rev. D 64, 116009 (2001); Int. J. Mod. Phys. A 18, 5475 (2003);

[8] A. V. Nesterenko and J. Papavassiliou, Phys. Rev. D 71, 016009 (2005); A. C. Aguilar, A. V. Nesterenko and J. Papavassiliou, J. Phys. G 31, 997 (2005). J. Phys. G 32, 1025 (2006) arXiv:hep-ph/0511215; A. V. Nesterenko, in Proceedings of the Ninth Workshop on Nonperturbative QCD, Paris, France, 2007 arXiv:0710.5878.

[9] A. I. Alekseev, Few Body Syst. 40, 57 (2006) arXiv:hep-ph/0503242.

[10] Y. Srivastava, S. Pacetti, G. Pancheri and A. Widom, in Proceedings of $e^{+} e^{-}$Physics at Intermediate Energies, SLAC, Stanford, CA, USA, 30 April - 2 May 2001, pp T19 arXiv:hep-ph/0106005.

[11] B. R. Webber, JHEP 9810, 012 (1998) arXiv:hep-ph/9805484.

[12] G. Cvetič and C. Valenzuela, J. Phys. G 32, L27 (2006) arXiv:hep-ph/0601050].

[13] G. Cvetič and C. Valenzuela, Phys. Rev. D 74, 114030 (2006) arXiv:hep-ph/0608256].

[14] G. M. Prosperi, M. Raciti and C. Simolo, Prog. Part. Nucl. Phys. 58, 387 (2007) arXiv:hep-ph/0607209].

[15] D. V. Shirkov and I. L. Solovtsov, Theor. Math. Phys. 150, 132 (2007) arXiv:hep-ph/0611229.

[16] G. Cvetič and C. Valenzuela, Braz. J. Phys. 38, 371 (2008) arXiv:0804.0872 [hep-ph]].

[17] K. A. Milton, I. L. Solovtsov, O. P. Solovtsova and V. I. Yasnov, Eur. Phys. J. C 14, 495 (2000) arXiv:hep-ph/0003030.

[18] M. A. Shifman, A. I. Vainshtein and V. I. Zakharov, Nucl. Phys. B 147, 385 (1979); Nucl. Phys. B 147, 448 (1979).

[19] G. Cvetič, R. Kögerler and C. Valenzuela, J. Phys. G 37, 075001 (2010) arXiv:0912.2466 [hep-ph]];

[20] G. Cvetič, R. Kögerler and C. Valenzuela, arXiv:1006.4199 [Unknown].

[21] G. Cvetič and H. E. Martínez, J. Phys. G 36, 125006 (2009) arXiv:0907.0033 [hep-ph]].

[22] S. Peris, Phys. Rev. D 74, 054013 (2006) arXiv:hep-ph/0603190.

[23] B. A. Magradze, arXiv:1005.2674 [Unknown].

[24] R. Barate et al. [ALEPH Collaboration], Eur. Phys. J. C 4, 409 (1998).

[25] S. Schael et al. [ALEPH Collaboration], Phys. Rept. 421, 191 (2005) arXiv:hep-ex/0506072.

[26] K. Ackerstaff et al. [OPAL Collaboration], Eur. Phys. J. C 7, 571 (1999) arXiv:hep-ex/9808019].

[27] S. Peris, M. Perrottet and E. de Rafael, JHEP 9805, 011 (1998) arXiv:hep-ph/9805442.

[28] E. Gardi, G. Grunberg and M. Karliner, JHEP 9807, 007 (1998) hep-ph/9806462 ; B. A. Magradze, arXiv:hep-ph/9808247

[29] D. S. Kourashev, arXiv:hep-ph/9912410 D. S. Kurashev and B. A. Magradze, Theor. Math. Phys. 135, 531 (2003) [Teor. Mat. Fiz. 135, 95 (2003)].

[30] B. A. Magradze, Few Body Syst. 40, 71 (2006) arXiv:hep-ph/0512374.

[31] Y. L. Dokshitzer, G. Marchesini and B. R. Webber, Nucl. Phys. B 469, 93 (1996) arXiv:hep-ph/9512336.

[32] G. Cvetič and C. Valenzuela, Phys. Rev. D 77, 074021 (2008) arXiv:0710.4530 [hep-ph]].

[33] K. G. Chetyrkin, B. A. Kniehl and M. Steinhauser, Phys. Rev. Lett. 79, 2184 (1997) arXiv:hep-ph/9706430.

[34] C. Amsler et al. [Particle Data Group], Phys. Lett. B 667, 1 (2008).

[35] A. P. Bakulev, Phys. Part. Nucl. 40, 715 (2009) (in Russian) arXiv:0805.0829 [hep-ph]]; A. P. Bakulev, S. V. Mikhailov and N. G. Stefanis, JHEP 1006, 085 (2010) arXiv:1004.4125|[Unknown]].

[36] G. Cvetič and R. Kögerler, Phys. Rev. D 63, 056013 (2001) arXiv:hep-ph/0006098.

[37] P. M. Stevenson, Phys. Rev. D 23, 2916 (1981).

[38] E. Braaten, Phys. Rev. Lett. 60, 1606 (1988); S. Narison and A. Pich, Phys. Lett. B 211, 183 (1988); E. Braaten, S. Narison, and A. Pich, Nucl. Phys. B 373, 581 (1992); A. Pich and J. Prades, JHEP9806, 013 (1998) hep-ph/9804462.

[39] K. G. Chetyrkin, A. L. Kataev and F. V. Tkachov, Phys. Lett. B 85, 277 (1979); M. Dine and J. R. Sapirstein, Phys. Rev. Lett. 43, 668 (1979); W. Celmaster and R. J. Gonsalves, Phys. Rev. Lett. 44, 560 (1980).

[40] S. G. Gorishnii, A. L. Kataev and S. A. Larin, Phys. Lett. B 259, 144 (1991); L. R. Surguladze and M. A. Samuel, Phys. Rev. Lett. 66, 560 (1991) [Erratum-ibid. 66, 2416 (1991)].

[41] P. A. Baikov, K. G. Chetyrkin and J. H. Kühn, Phys. Rev. Lett. 101, 012002 (2008) arXiv:0801.1821 [hep-ph]].

[42] M. Neubert, Phys. Rev. D 51, 5924 (1995) hep-ph/9412265. 
[43] M. Neubert, hep-ph/9502264

[44] G. Cvetič, M. Loewe, C. Martínez and C. Valenzuela, arXiv:1005.4444 [Unknown].

[45] A. Deur et al., Phys. Rev. D 78, 032001 (2008) arXiv:0802.3198 [nucl-ex]].

[46] A. L. Kataev, Phys. Rev. D 50, R5469 (1994) arXiv:hep-ph/9408248]; JETP Lett. 81, 608 (2005) [Pisma Zh. Eksp. Teor. Fiz. 81, 744 (2005)] arXiv:hep-ph/0505108; Mod. Phys. Lett. A 20, 2007 (2005) arXiv:hep-ph/0505230.

[47] R. S. Pasechnik, D. V. Shirkov and O. V. Teryaev, Phys. Rev. D 78, 071902 (2008) arXiv:0808.0066 [hep-ph]].

[48] R. S. Pasechnik, D. V. Shirkov, O. V. Teryaev, O. P. Solovtsova and V. L. Khandramai, Phys. Rev. D 81, 016010 (2010) arXiv:0911.3297.

[49] S. G. Gorishny and S. A. Larin, Phys. Lett. B 172, 109 (1986); E. B. Zijlstra and W. Van Neerven, Phys. Lett. B 297, 377 (1992); S. A. Larin and J. A. M. Vermaseren, Phys. Lett. B 259, 345 (1991).

[50] A. L. Kataev and V. V. Starshenko, Mod. Phys. Lett. A 10, 235 (1995) hep-ph/9502348.

[51] D. J. Broadhurst and A. L. Kataev, Phys. Lett. B 315, 179 (1993) hep-ph/9308274. 